\title{
Volatility Spillovers and Correlations between Oil Prices and Stock Sectors in Turkey: Implications on Portfolio Hedging and Diversification Opportunities
}

Vasıf ABİOĞLU (https://orcid.org/0000-0002-8217-0702), Department of Economics, Aksaray University, Turkey; e-mail: vabiyev@aksaray.edu.tr

\section{Türkiye'de Petrol Fiyatları ve Sanayi Sektörleri Arasında Volatilite Yayılımı ve Korelasyon: Riskten Korunma ve Portföy Çeşitlendirme Üzerine Etkileri}

\begin{abstract}
This study investigates volatility spillover effects as well as hedging and diversification opportunities between sectoral stock returns and world crude oil prices in Turkey using the weekly closing prices of the BIST 100 and twenty-three sectoral stock indices for the period 2002-2018. DCC modelling is employed to investigate volatility spillovers between sectoral stock returns and oil prices. Findings reveal significant volatility spillovers from the oil market to the BIST 100 and twelve stock sectors. Furthermore, optimal hedge ratios, optimal portfolio weights, hedging effectiveness, diversification effectiveness and risk-adjusted returns of oil-stock portfolios are computed and compared. The results indicate that diversification is a more effective strategy than hedging in terms of risk (variance) reductions and risk-adjusted returns in the Turkish stock market.
\end{abstract}

Keywords

Volatility Spillover, Conditional Correlations, Hedge Ratio, Hedging Effectiveness, Optimal Weight, Diversification Effectiveness.

JEL Classification Codes : $\quad$ C58, G11, G17, G32, Q43.

$\ddot{O} z$

Bu çalışmada, 2002-2018 dönemi için haftalık veriler kullanılmakla, dünya ham petrol fiyatları ile Türkiye'de BIST 100 ve 23 sanayi sektörü getiri oranları arasındaki volatilite yayılmaları, riskten korunma ve portföy çeşitlendirme stratejileri incelenmektedir. DCC modeli ile elde edilen volatilite yayılmaları tahmin sonuçlara göre, petrol fiyatlarından BIST 100 ve 12 sanayi sektörüne anlamlı volatilite geçişleri söz konusudur. Ayrıca, çalışmada petrol-sektör portföyü için optimal riskten korunma oranları, optimal portföy ağırlık oranları, riskten korunma etkinliği, portföy çeşitlendirme etkinliği ve risk-ayarlı getiri oranları hesaplanmaktadır. Tahmin sonuçlarına göre, risk (varyans) azalışı ve risk-ayarlı getiri oranları açısından portföy çeşitlendirme stratejisi riskten korunma stratejisine oranla daha etkin strateji olmaktadır.

Anahtar Sözcükler : Volatilite Yayılımı, Koşullu Korelasyon, Riskten Korunma Oranı, Riskten Korunma Etkinliği, Optimal Portföy Ağırlığı, Çeşitlendirme Etkinliği. 
Abioğlu, V. (2021), "Volatility Spillovers and Correlations between Oil Prices and Stock Sectors in Turkey: Implications on Portfolio Hedging and Diversification Opportunities", Sosyoekonomi, 29(47), 79-106.

\section{Introduction}

The role of oil in an economy is crucial not only for macroeconomic indicators but also for financial markets. Research on the relationship between oil price shocks and stock markets has garnered much attention in recent years. The relationship between oil price changes and stock prices can be explained using an equity pricing model. In this type of model, the price of equity at any point in time is equal to the expected present value of discounted future cash flows (Jones \& Kaul, 1996; Huang et al., 1996; Basher \& Sadorsky, 2006; Park \& Ratti, 2008). Because oil is a main input of production, increases in oil prices lead to high production costs, which dampen cash flows and reduce stock prices. In addition, high oil prices often generate inflationary pressures which central banks can control by raising interest rates. Higher interest rates make bonds more attractive than stocks, leading to a fall in stock prices. Overall, the impact of rising oil prices on stock prices is expected to be negative.

Oil price volatility has drawn a lot of attention in recent years due to its effect on stock markets. The volatility of price changes can be an accurate measure of the rate of information flow in a stock market (Ross, 1989: 2-3). It is possible that no significant effects of oil prices are observable in stock prices, but oil price volatility - the rate of information flow in the oil market - may lead to volatility in the stock market (Huang et al., 1996: 2-5). Sharp changes in oil price, whatever the reason, may reduce aggregate output temporarily because they curtail investment due to increasing uncertainty or induce costly resource reallocation (Guo \& Kliesen, 2005: 669-670). Increased volatility in oil prices can affect the present value of the discounted stream of dividend payments, by increasing uncertainty regarding product demand and doing the same for future return on investment (Park \& Ratti, 2008: 2601).

Multivariate generalized autoregressive conditional heteroscedasticity (MGARCH) models have been found very useful in studying volatility spillover effects between asset returns (Kroner \& Sultan, 1993; Kroner \& Ng, 1998; Ling \& McAleer, 2003; Capiello et al., 2006; Ku et al., 2007; Malik \& Hammoudeh, 2007; Malik \& Ewing 2009; McAleer et al., 2009; Hammoudeh et al, 2010; Chang et al., 2011; Arouri et al., 2011, 2012; Sadorsky, 2012; Mensi et al., 2013; Basher \& Sadorsky, 2016). The main advantages of the MGARCH approach are that it allows for the investigation of the following: shock transmission, the dynamics of conditional volatility, conditional correlations and volatility spillovers between asset returns. Furthermore, empirical findings can be used to compute the optimal hedge ratios, optimal weights and hedging effectiveness of an oil-stock portfolio. Several papers have examined volatility spillovers between oil and stock prices from a sectoral perspective by using different specifications of MGARCH models (Malik \& Ewing, 2009; Arouri et al., 2011, 2012; Sadorsky, 2012; Mensi et al., 2014; Lin et al., 2014; Caporale et al., 2015; Basher \& Sadorsky, 2016; Kang et al., 2017; Al-Maadid et al., 2017; Ahmad et al., 2018; Kirkulak-Uludag \& Safarzadeh, 2018; etc).

There are many studies that investigate the impact of crude oil prices on Turkish stock markets and that focus primarily on the cointegration relationships between oil prices and 
Abioğlu, V. (2021), "Volatility Spillovers and Correlations between Oil Prices and Stock Sectors in Turkey: Implications on Portfolio Hedging and Diversification Opportunities", Sosyoekonomi, 29(47), 79-106.

stock market and sectors. (Al-Fayoumi, 2009; İşcan, 2010; Güler, Tunç \& Orçun, 2010; Kapusuzoglu, 2011; Sayılgan \& Süslü, 2011; Unlu \& Topcu, 2012; Aktaş \& Akdağ, 2013; Şener et al., 2013; Abdioğlu \& Değirmenci, 2014; Eyüboğlu \& Eyüboğlu, 2016; Büberkökü, 2017). Most of these studies find a long-term cointegration relationship between crude oil prices and stock market (sectors) and unidirectional causality running from oil prices to the stock market for different periods. Unfortunately, very few studies analyze volatility spillover between oil prices and Turkish sectoral stock returns. Soytas \& Oran (2011) employ a causality-in-variance test to investigate volatility spillovers from the oil market to the BIST 100 and electricity stock sector in Turkey by using daily data for the period 20032007. They have found that there is volatility spillover from world oil spot markets to electricity stock index returns but not to the BIST 100 index returns in Turkey. On the other hand, Gencer \& Demiralay (2014) employ a BEKK-GARCH model to examine the volatility spillover between oil and five major sector indices (BIST 100; Banking; Chemical, Petrol \& Plastic; Industrials; Services) in Turkey using daily data for the period 2005-2013. Their findings indicate unidirectional volatility spillovers from the oil market to all of the examined sectors. For the Banking, Industrial, and Services sectors, the results document significant shock transmissions from the oil market. Gönüllü et al. (2015) employ a multifactor model to examine the impact of crude oil price on the Chemical, Petrol \& Plastic sector using daily data for the period 2003-2012. The findings indicate that crude oil prices positively impact sector returns.

In this study, firstly, a DCC-VARMA-GARCH model is employed to investigate volatility spillovers and conditional correlations between Turkish sectoral stock returns and world crude oil prices, as the DCC approach allows better estimation of spillovers and conditional correlations between oil and stock returns. Secondly, hedging and diversification strategies are investigated in the Turkish stock market. For this purpose, optimal hedge ratios, optimal portfolio weights, hedging effectiveness, diversification effectiveness and risk-adjusted returns of hedged and diversified oil-stock portfolios are computed and compared using four different multivariate GARCH models (CCC-VARMA-GARCH, CCC-VARMA-AGARCH, DCC-VARMA-GARCH and ADCC-VARMA-GARCH models). The results indicate that DCC- and ADCC-VARMA-GARCH models are the best in terms of variance reduction in hedged portfolios for all sectors. Additionally, portfolio diversification has been found to be a more effective strategy than portfolio hedging in terms of variance reductions and risk-adjusted returns of oil-stock portfolios in Turkish stock markets. Essentially, hedging and diversification strategies are mutually exclusive. Investors can hedge or diversify their portfolios in order to minimize risk without reducing expected return. Financial markets allow investors to create diversified or hedged portfolios. If investors have insufficient capital to diversify their portfolios, they can hedge their portfolios against unfavorable price changes by taking offsetting positions. A hedging strategy protects an asset against a certain risk of another asset if these two assets are correlated with each other. Hence, the magnitude of hedging is positively related to the magnitude of correlations between assets. However, in a diversification strategy, the risk of one asset can be used to offset the risk of other assets particularly in cases of negatively correlated or uncorrelated assets. That is, negative as well as close-to-zero correlations between crude oil and sector returns provide better diversification opportunities for stock sectors. To the best of our 
knowledge, this is the first study to examine volatility spillover between oil prices and stock returns for the BIST 100 and twenty-three sector indices. Furthermore, this study is the first to examine hedge ratios between oil and stock returns, optimal portfolio weights for oilstock portfolios, hedging and diversification effectiveness, and to compare the risk-adjusted returns of hedged and diversified portfolios with risk-adjusted returns of unhedged portfolios. In these respects, this study aims to contribute to the literature by analysing the volatility spillover from the oil market to disaggregated stock returns, and to discuss hedging strategies.

The outline of the study is structured as follows: Section 2 develops econometric methodology. In this section, two newly developed conditional volatility models, the consistent DCC and consistent ADCC models, are described. Section 3 describes data and presents the empirical results, and Section 4 provides concluding remarks.

\section{Econometric Methodology}

The VAR-GARCH model developed by Ling \& McAleer (2003) has been found useful for studying the dynamics of conditional volatility, shock transmissions and the volatility spillovers between stock sector returns and oil prices. For each pair of stock returns (sector or market returns) and oil returns, the bivariate $\operatorname{VAR}(1)-\operatorname{GARCH}(1,1)$ model of Ling $\&$ McAleer (2003) has the following specification for the conditional mean:

$$
r_{t}=\alpha+\beta r_{t-1}+\varepsilon_{t}, \varepsilon_{t}=v_{t} \sqrt{H_{t}} \text { and } v_{t} \sim \text { i.i.d. }(0,1)
$$

where $r_{t}=\left[r_{S, t}, r_{O, t}\right]^{\prime}$ is the vector of returns for stock and oil price indices, respectively. $\beta$ is the $2 \mathrm{X} 2$ matrix of coefficients of the form $\beta=\left[\begin{array}{cc}\beta_{1} & 0 \\ 0 & \beta_{2}\end{array}\right] . \varepsilon_{t}=\left[\varepsilon_{S, t}, \varepsilon_{O, t}\right]^{\prime}$ is the vector of error terms of the conditional mean equations for stock and oil returns, respectively. If stock returns exhibit a high degree of persistency, then additional AR coefficients can be added to equation (1) in order to eliminate the autocorrelation problem in error terms. $v_{t}=\left[v_{S, t}, v_{O, t}\right]^{\prime}$ is the vector of independently and identically distributed (i.i.d.) random errors, and $H_{t}=\left[\begin{array}{cc}h_{S, t} & h_{S O, t} \\ h_{S O, t} & h_{O, t}\end{array}\right]$ is the $2 \mathrm{X} 2$ conditional variance-covariance matrix of stock and oil returns.

In Ling \& McAleer's (2003) CCC-VARMA-GARCH(1,1) model (hereinafter CCC model), the $h_{S, t}, h_{O, t}$ and $h_{S O, t}$ equations are defined as follows:

$$
\begin{aligned}
& h_{S, t}=c_{S}+a_{S}\left(\varepsilon_{S, t-1}\right)^{2}+b_{S} h_{S, t-1}+a_{O}\left(\varepsilon_{O, t-1}\right)^{2}+b_{O} h_{O, t-1} \\
& h_{O, t}=c_{O}+a_{O}\left(\varepsilon_{O, t-1}\right)^{2}+b_{O} h_{O, t-1}+a_{S}\left(\varepsilon_{S, t-1}\right)^{2}+b_{S} h_{S, t-1}
\end{aligned}
$$




$$
h_{S O, t}=\rho_{S O} \times \sqrt{h_{S, t}} \times \sqrt{h_{O, t}}
$$

As seen from equations (2) and (3), volatility spillover across the oil and stock markets over time is governed through the lagged cross values of error terms $\left(\varepsilon_{0, t-1}\right)^{2}$ and $\left(\varepsilon_{S, t-1}\right)^{2}$, which capture the impact of direct effects of shock transmission, as well as lagged conditional volatilities $h_{O, t-1}$ and $h_{s, t-1}$. To guarantee the stationarity of the series in the $H_{t}$ variance-covariance matrix, the roots of the equation $|I-A L-B L|=0$ must be outside the unit circle, where $L$ is the lag polynomial and $A=\left[\begin{array}{ll}a_{S 1} & a_{S 2} \\ a_{O 1} & a_{O 2}\end{array}\right]$ and $B=\left[\begin{array}{ll}b_{S 1} & b_{S 2} \\ b_{O 1} & b_{O 2}\end{array}\right]$. $\rho_{S O}$ is the constant conditional correlation in the conditional covariance equation (4). As specified above, equations (2) and (3) allow long-run volatility persistence as well as shock and volatility transmissions between the oil and stock markets. Ling and McAleer (2003) show that the QMLE (quasi-maximum-likelihood estimation) method consistently estimates the parameters of equations (1)-(4).

The CCC model assumes that negative and positive shocks of equal magnitude have identical impacts on the conditional variance. McAleer et al. (2009) extended the CCC model to accommodate the asymmetric impacts shocks on the conditional variance, and proposed the CCC-VARMA-AGARCH $(1,1)$ (hereinafter CCC-AGARCH) specification of the conditional variance as follows:

$$
\begin{aligned}
& h_{S, t}=c_{S}+a_{S}\left(\varepsilon_{S, t-1}\right)^{2}+d_{S} I_{t-1}\left(\varepsilon_{S, t-1}\right)^{2}+b_{S} h_{S, t-1}+a_{O}\left(\varepsilon_{O, t-1}\right)^{2}+b_{O} h_{O, t-1} \\
& h_{O, t}=c_{O}+a_{O}\left(\varepsilon_{O, t-1}\right)^{2}+d_{O} I_{t-1}\left(\varepsilon_{O, t-1}\right)^{2}+b_{O} h_{O, t-1}+a_{S}\left(\varepsilon_{S, t-1}\right)^{2}+b_{S} h_{S, t-1}
\end{aligned}
$$

where $I_{t-1}=1$ if $\varepsilon_{S, t-1}<0$ and $\varepsilon_{O, t-1}>0$, and zero otherwise. The dummy variable indicates that negative shocks to sectoral stock returns and positive shocks to oil prices increase volatilities in stock sectors and oil market, respectively. The structural and statistical properties of the models, including the necessary and sufficient conditions for stationarity and ergodicity of the CCC and CCC-AGARCH models, are explained in detail in Ling \& McAleer (2003) and McAleer et al. (2009), respectively.

Engle (2002) remedies the restrictive assumption of the constant conditional correlations by allowing the conditional correlation matrix to vary over time. The assumption of constant conditional correlation may seem unrealistic. In order to make the conditional correlation matrix time-dependent, Engle (2002) proposed a dynamic conditional correlation (DCC) model, which is estimated in two steps. In the first step, the GARCH parameters are estimated. In the second step, the time-varying conditional correlation matrix $\left(P_{t}\right)$ is estimated. 


$$
H_{t}=D_{t} P_{t} D_{t}
$$

where $D_{t}=\operatorname{diag}\left(\sqrt{h_{S, t}}, \sqrt{h_{O, t}}\right)$ and $P_{t}$ is the time-varying conditional correlation matrix. $h_{S, t}$ and $h_{O, t}$ are represented by equations (2) and (3) respectively. Hence, this model is named the DCC-VARMA-GARCH $(1,1)$ model (hereinafter the DCC model). In this model, the time-varying conditional correlation matrix is defined as

$$
\begin{aligned}
& P_{t}=Q_{t}^{*-1} Q_{t} Q_{t}^{*-1} \\
& Q_{t}=\left(1-\theta_{1}-\theta_{2}\right) \bar{Q}+\theta_{1} z_{t-1} z_{t-1}^{\prime}+\theta_{2} Q_{t-1}, \theta_{1}, \theta_{2}>0 \text { and } \theta_{1}+\theta_{2}<1
\end{aligned}
$$

where $Q_{t}^{*}=\operatorname{diag}\left\{\sqrt{q_{S, t}}, \sqrt{q_{O, t}}\right\}, z_{t}=\left[\frac{\varepsilon_{S, t}}{\sqrt{h_{S, t}}}, \frac{\varepsilon_{o, t}}{\sqrt{h_{O, t}}}\right]^{\prime}$ and $Q_{t}$ is a positive definite symmetric matrix. $\bar{Q}$ is $2 \mathrm{X} 2$ unconditional correlation matrix of the standardized residuals $\left(z_{t}\right)$. Under the DCC specification, time-varying conditional correlation coefficients and conditional covariance equations are defined as

$$
\begin{aligned}
& \rho_{S O, t}=\frac{q_{S O, t}}{\sqrt{q_{S, t} q_{O, t}}} \\
& h_{S O, t}=\rho_{S O, t} \times \sqrt{h_{S, t}} \times \sqrt{h_{O, t}}
\end{aligned}
$$

However, Aielli (2013) shows that the estimation of $Q_{t}$ in this way is inconsistent in the sense that $E\left(z_{t} z_{t}^{\prime}\right) \neq E\left(Q_{t}\right)$ and he proposes the following consistent DCC ( $c$ DCC) model:

$$
Q_{t}=\left(1-\theta_{1}-\theta_{2}\right) \bar{Q}^{*}+\theta_{1}\left(Q_{t-1}^{*} z_{t-1} z_{t-1}^{\prime} Q_{t-1}^{*}\right)+\theta_{2} Q_{t-1}
$$

where $z_{t-1}$ is scaled by the diagonal elements of $Q_{t-1}^{*}$, and $\overline{\boldsymbol{Q}}^{*}$ is the unconditional correlation matrix of $Q_{t}^{*} z_{t}$.

Since the DCC model fails to capture asymmetric effects, Capiello, Engle and Sheppard (2006) introduce asymmetric effects in dynamic conditional correlations (ADCC). Asymmetric effects play a central role in revealing the role of negative stock returns (or positive oil price changes) during unstable periods compared to the stable periods. $Q_{t}$ in the ADCC-VARMA-GARCH(1,1) model (hereinafter referred to as the ADCC model) is specified as follows:

$$
Q_{t}=\left(1-\theta_{1}-\theta_{2}\right) \bar{Q}-\phi \bar{N}+\theta_{1} z_{t-1} z_{t-1}^{\prime}+\theta_{2} Q_{t-1}+\phi n_{t-1} n_{t-1}^{\prime}
$$


where the coefficient $\phi$ shows asymmetric effect, $n_{t}=I\left[z_{1 t}<0, z_{2 t}>0\right] \otimes z_{t}$ where $\otimes$ is the Hadamard product, and $\bar{N}=T^{-1} \sum_{1}^{T} n_{t} n_{t}^{\prime}$. Here, $I[\cdot]=1$ if $z_{1 t}=\frac{\varepsilon_{S, t}}{\sqrt{h_{S, t}}}<0$ and $z_{2 t}=\frac{\varepsilon_{O, t}}{\sqrt{h_{O, t}}}>0$, and zero otherwise. This means that ADCC helps explain the changes in conditional correlation during negative shocks to sectoral stock returns and positive shocks to oil prices. As in the DCC model, in equation (12) $z_{t-1}$ can be scaled by the diagonal elements of $Q_{t-1}^{*}$ in order to get consistent ADCC. So, $z_{t-1}$ is scaled by $Q_{t-1}^{*}$ in equation (12) in order to get consistent ADCC as shown below.

$$
Q_{t}=\left(1-\theta_{1}-\theta_{2}\right) \bar{Q}^{*}-\phi \bar{N}+\theta_{1}\left(Q_{t-1}^{*} z_{t-1} z_{t-1}^{\prime} Q_{t-1}^{*}\right)+\theta_{2} Q_{t-1}+\phi n_{t-1} n_{t-1}^{\prime}
$$

All MGARCH models described above are estimated by Quasi-Maximum Likelihood estimation (QMLE) using the BFGS algorithm. $t$ statistics of parameters are calculated using robust estimation of the covariance matrix. The DCC model is used to estimate volatility dynamics and conditional correlations between sectoral stock returns and oil prices, while the CCC, CCC-AGARCH and ADCC models are used in particular to compare the results of hedging effectiveness and portfolio diversifications.

\section{Data and Empirical Results}

\subsection{Data}

Our sample data cover the BIST 100 and twenty-three industrial sector indices. All stock data have been collected from the Borsa Istanbul Equity Market Daily Closing Prices and Trade Volumes database on a weekly basis. More precisely, Friday closing prices have been chosen for each week. For the crude oil market, the Brent crude oil spot price was taken from the Energy Information Administration which represents the oil market. The oil price data were expressed in Turkish Lira using \$/TL exchange rates obtained from the official website of the Central Bank of Turkey. Since weekly data seem to capture the dynamic interaction of oil and stock prices better than daily and monthly data, weekly data are employed in the analysis for the period from January 11, 2002 to December 28, 2018. Oil and stock returns are calculated as $r_{i t}=\ln \left(\frac{P_{t}}{P_{t-1}}\right) * 100^{1}$.

Table 1 reports the main descriptive statistics and stochastic properties of the return series. As seen from the table, the mean value of the market and all sector returns are positive in the analyzed period. On average, mean returns of some sectors are realized higher than the oil price return. Further, average returns of the overall stock market (BIST 100), oil market and 12 other sectors (Food \& Beverage; Chemical, Petrol \& Plastic; Basic Metal; Metal Products \& Machinery; Insurance; Services; Industrials; Technology; Non-Metal

1 Kalman smoothing recursions are used to deal with the missing data points on public holidays. 
Abioğlu, V. (2021), "Volatility Spillovers and Correlations between Oil Prices and Stock Sectors in Turkey: Implications on Portfolio Hedging and Diversification Opportunities", Sosyoekonomi, 29(47), 79-106.

Mineral Products; Wholesale \& Retail Trade; Leasing \& Factoring; and Transportation) are significantly different from zero. The skewness statistic is positive for Banks, Securities Investment Trusts, Leasing \& Factoring and Tourism sectors; zero for Wholesale \& Retail Trade, Financials, oil market and Real Estate Investment Trusts (REIT); and negative for all other sectors. This means that Banks, Securities Investment Trusts, Leasing \& Factoring and Tourism sectors have extreme gains (longer right tails), and all other sectors have extreme losses (longer left tails) except for Wholesale \& Retail Trade, Financials, oil market and REITs. Moreover, the excess kurtosis statistic is significant at $1 \%$ level for all sectors, which means that all sectors have large amounts of extreme values in their tails. Normality conditions $(J B)$ are rejected for all sectors at $1 \%$ significance level. Ljung-Box serial correlation $(Q(10))$ test results indicate that oil and fifteen of twenty-three sectoral return series have serial correlations. For the squared returns, all variables exhibit significantly high serial correlation at lags 10. Finally, strong evidence of ARCH effect is found for all sectoral stock returns except for Technology².

Unconditional correlation coefficients between stock returns and oil returns, reported in Table 1, are generally weak for all sectors. The sectors that have high positive correlations with the oil market are Chemical, Petrol \& Plastic (0.070), Basic Metal (0.060) and Industrials (0.055), while the sectors that have lowest positive correlations with the oil market are Technology (0.001), Wholesale \& Retail Trade (0.007) and Real Estate Investment Trusts (0.008). Negative unconditional correlations were found for eight sectors: Banks (-0.0001), Information Technology (-0.047), Telecommunication (-0.011), Services (-0.012), Non-Metal Mineral Products (-0.012), Leasing \& Factoring (-0.013), Transportation (-0.050) and Tourism (-0.052). On the other hand, all sectors have high positive correlations with the BIST 100.

\subsection{Volatility Spillovers and Dynamic Conditional Correlations}

The DCC model is employed to examine volatility dynamics and conditional correlations between the oil and stock sectors. The estimated results of the DCC model for the BIST 100 and twenty-three sectors are presented in Table $2^{3}$. The estimation results show that estimated AR coefficients of mean equations are statistically significant for ten sectors: Information Technology, Textile \& Leather, Food \& Beverage, Telecommunication, Insurance, Wholesale \& Retail Trade, Non-Oil Mineral Products, Transportation, Real Estate Investment Trusts (REIT) and Tourism. Among these sectors, the Wholesale \& Retail Trade, Non-Metal Mineral Products, Transportation, REIT and Tourism sectors indicate positive high-order autoregressive processes in their mean equations ${ }^{4}$. This means that these

2 Technology sector indicates strong ARCH effects for the lags higher than 10.

$3 \operatorname{VAR}(p)$ and $\operatorname{ARMA}(p, q)$ models were also employed for the mean equations of all sectors. However, it is found that the AR(p) specification best fits the mean equations of all sectors. Further, it is found that among twenty four sectors, the AR(3) specification for Wholesale \& Retail Trade, the AR(2) specification for Non-Metal Mineral Products, Transportation, REIT and Tourism and the AR(1) specification for the other nineteen sectors best fit the data in terms of eliminating autocorrelations in the residuals.

4 The mean equation of Wholesale and Retail Trade also includes two dummy variables for huge positive and negative outliers in 2008.11.21 and 2008.11.28 in order to cope with the remaining heteroskedasticity and 
Abioğlu, V. (2021), "Volatility Spillovers and Correlations between Oil Prices and Stock Sectors in Turkey: Implications on Portfolio Hedging and Diversification Opportunities", Sosyoekonomi, 29(47), 79-106.

sectors exhibit higher degrees of persistency in their return series relative to other sectors which exhibit AR(1) processes in the analysed period. High degrees of persistency in return series can be attributed to the high growth potential of the Turkish economy during this period. After the adoption of an inflation targeting regime together with a free-floating exchange rate regime and some reform programs in the early 2000s, the Turkish economy entered a high-growth phase which boosted the returns of some key industries like trade, construction, tourism and transportation. However, for the other thirteen sectors, stock returns did not exhibit any autoregressive processes, which indicates some evidence of unpredictability in the stock prices of those sectors. This means that the weak-form of informational efficiency is accepted for the majority of sectors in the Turkish stock market.

Estimates of own conditional ARCH and GARCH coefficients in the conditional variance equations are positive and statistically significant at conventional significance levels for the majority of sectors. The sum of own ARCH and GARCH coefficients ( $a_{S}$ and $b_{S}$ ) is less than unity, implying that the volatility process is mean reverting for all sectors. Own conditional GARCH coefficients, which measure volatility persistence, appear to be significant at $1 \%$ significance level for all sectors. Further, among all sectors, Securities Investment Trusts, Leasing \& Factoring, and Insurance have the highest volatility persistence, while Food \& Beverage, Electricity, and Tourism have the lowest volatility persistence. Own conditional ARCH effects, which measure own shock dependence, are statistically significant for all sectors except for Insurance and REITs. Further, according to the estimation results, Tourism and Electricity have the highest own shock dependence, while Wholesale \& Retail Trade, Leasing \& Factoring, and Securities Investment Trusts have the lowest own shock dependence.

The analysis of volatility transmission between the oil and stock sectors shows significant volatility spillovers from the oil market. According to the estimation results, shocks in the oil market significantly increase conditional volatilities of the BIST 100 and ten sectors: Banks; Information Technology; Textile \& Leather; Holding \& Investments; Telecommunication; Chemical, Petrol \& Plastic; Metal Products \& Machinery; Financials; Services and Industrials ${ }^{5}$. Among these sectors, the highest oil shock transmission effects are found in Textile \& Leather (0.038) and Industrials (0.026), while the lowest oil-shock

autocorrelation problems in the residuals of the equation. Further, the variance equation of Leasing \& Factoring includes a dummy variable for huge oscillations in the return series in the period of 2018.02.022018.12.28 in order to cope with the remaining autocorrelation and heteroskedasticity problems in the residuals.

5 The CCC model has also been employed and has found similar shock transmissions from oil to stock returns for the BIST 100 and the same ten sectors mentioned above. However, any significant constant conditional correlation coefficients were not found in the CCC model except for the following sectors: Information \& Technology; Chemical, Petrol \& Plastic; Transportation; and Tourism. In view of space limitations, the estimation results of the CCC model were not presented in this study. The estimation results and their test statistics are available upon request. Further, the asymmetric versions of the CCC and DCC models, the CCCAGARCH and ADCC models, have been employed to investigate volatility spillovers and conditional correlations. However, the asymmetric coefficients of these models were found to be statistically insignificant for most sectors. Due to space limitations, the estimation results of the CCC-AGARCH and ADCC models are available on request. 
Abioğlu, V. (2021), "Volatility Spillovers and Correlations between Oil Prices and Stock Sectors in Turkey: Implications on Portfolio Hedging and Diversification Opportunities", Sosyoekonomi, 29(47), 79-106.

transmission effects are found in Metal Products \& Machinery (0.010) and Holding \& Investments (0.013). The estimation results also indicate that past volatility persistence in the oil market significantly increases conditional volatilities of Food \& Beverage and Basic Metal. These volatility relationships are not surprising. Since rising oil prices negatively affect producers' profitability in the Food \& Beverage and Basic Metal sectors, high oil prices tend to increase the conditional volatilities of stock returns in these sectors. On the other hand, the past conditional volatility of oil significantly decreases the conditional volatility of the Chemical, Petrol \& Plastic sector. Since this sector includes major oil producing companies in Turkey, this finding indicates that high oil prices positively affect oil corporations' performances and thus decrease the conditional volatility of this sector.

As for the opposite direction, any significant volatility spillover effects from stock sectors to the oil market have not been found except for in Food \& Beverage. Past volatility and shocks in stock prices of Food \& Beverage significantly increase conditional volatility of oil prices that also reflect the effects of changes in exchange rate. As a result, with the exception of Food \& Beverage, oil market volatility behaves independently from shocks and volatilities of stock sectors in the Turkish economy.

Regarding the estimation of dynamic conditional correlations, the results in Table 2 indicate that estimated $\theta_{1}$ and $\theta_{2}$ coefficients are each positive and statistically significant for the BIST 100 and thirteen stock sectors: Banks; Information Technology; Food \& Beverage; Chemical, Petrol \& Plastic; Basic Metal; Metal Products \& Machinery; Insurance; Electricity; Financials; Industrials; Non-Metal Mineral Products; Transportation and Tourism. The sum of these coefficients are less than unity, meaning that the dynamic conditional correlations are mean reverting. Figure 1 shows the evolution of the dynamic conditional correlations between the oil and stock sectors. It is clear that there are significant fluctuations in the conditional correlations over time. One can see that the time path of dynamic conditional correlations for Industrials are similar to its sub-sectors of Food \& Beverage, Chemical, Petrol \& Plastic and Metal Products \& Machinery. Figure 1 also shows that the dynamic conditional correlations between sectors and oil are generally at low levels. In theory, close-to-zero correlations between two asset returns provide better opportunities for portfolio diversifications over these assets than assets with relatively high positive correlations. Particularly, in the case of uncorrelated assets, the risk of one asset can be used to offset the risk of another without reducing the expected return. Further, as will be shown in the next section, periods of negative correlations between oil and sectoral stock returns provide better opportunities for portfolio diversification, since negative correlations require long positions in both oil and sector stocks.

Diagnostic test results for standardized residuals show that autocorrelation and ARCH effects are eliminated, and non-normality is greatly reduced for all stock sectors in Table 2. Diagnostic tests of standardized residuals and standardized residuals squared show no evidence of autocorrelation at conventional significance levels. In addition, ARCH test results indicate no evidence of serial $\mathrm{ARCH}$ effects at conventional significance levels for all sectors. 
Table: 1

Descriptive Statistics of Return Series

\begin{tabular}{|c|c|c|c|c|c|c|c|c|c|c|c|}
\hline Sectors & $\mathrm{T}$ & Mean & Std. Er. & Sk. & Ex. Ku. & $J B$ & $Q(10)$ & $Q s q(10)$ & $\mathrm{ARCH}(10)$ & $\rho_{m i}$ & $\rho_{o i}$ \\
\hline Oil & 885 & $0.254^{* * *}$ & 4.142 & -0.049 & $1.894^{*}$ & $132.701^{*}$ & $63.666^{*}$ & $103.976^{*}$ & $6.709^{*}$ & 0.025 & 1.000 \\
\hline BIST 100 & 885 & $0.214^{* * *}$ & 3.851 & $-0.192^{* *}$ & $4.070^{*}$ & $616.315^{*}$ & 13.410 & $68.118^{*}$ & $5.560^{*}$ & 1.000 & 0.025 \\
\hline Banks & 885 & 0.189 & 5.037 & $0.136^{* * *}$ & $4.359^{*}$ & $703.474^{*}$ & 11.604 & $31.854^{*}$ & $2.423^{*}$ & 0.951 & -0.0001 \\
\hline Information Technology & 885 & 0.065 & 4.381 & $-0.265^{*}$ & $4.385^{*}$ & $719.543^{*}$ & $19.879^{* *}$ & $34.531^{*}$ & $3.432^{*}$ & 0.712 & -0.047 \\
\hline Textile \& Leather & 885 & 0.186 & 3.705 & $-0.916^{*}$ & $4.188^{*}$ & $770.808^{*}$ & 14.170 & $44.122^{*}$ & $4.425^{*}$ & 0.710 & 0.019 \\
\hline Food \& Beverage & 885 & $0.217^{* * *}$ & 3.709 & $-0.294^{*}$ & $2.116^{*}$ & $177.903^{*}$ & $28.287^{*}$ & $62.455^{*}$ & $5.052^{*}$ & 0.677 & 0.025 \\
\hline Holding \& Investment & 885 & 0.186 & 4.222 & $-0.304^{*}$ & $4.356^{*}$ & $713.393^{*}$ & 13.151 & $97.737^{*}$ & $7.872^{*}$ & 0.934 & 0.049 \\
\hline Telecommunication & 885 & 0.174 & 4.551 & $-0.163^{* *}$ & $2.572^{*}$ & $247.880^{*}$ & $16.582^{* * *}$ & $115.399^{*}$ & $5.776^{*}$ & 0.653 & -0.011 \\
\hline Wood, Paper \& Printing & 885 & 0.170 & 4.016 & $-0.362^{*}$ & $2.494^{*}$ & $248.833^{*}$ & $16.237^{* * *}$ & $31.827^{*}$ & $2.637^{*}$ & 0.739 & 0.025 \\
\hline Chemical, Petrol \& Plastic & 885 & $0.252^{* *}$ & 3.819 & $-0.271^{*}$ & $4.019^{*}$ & $606.510^{*}$ & 12.404 & $81.309^{*}$ & $7.277^{*}$ & 0.812 & 0.070 \\
\hline Basic Metal & 885 & $0.376^{* *}$ & 4.763 & $-0.441^{*}$ & $2.786^{*}$ & $314.921^{*}$ & 13.791 & $227.718^{*}$ & $13.615^{*}$ & 0.740 & 0.060 \\
\hline Metal Products \& Machinery & 885 & $0.238^{* * *}$ & 4.006 & $-0.564^{*}$ & $4.655^{*}$ & $846.178^{*}$ & $26.116^{*}$ & $148.795^{*}$ & $11.408^{*}$ & 0.854 & 0.038 \\
\hline Insurance & 885 & $0.287^{* *}$ & 4.326 & $-0.659^{*}$ & $6.268^{*}$ & $1512.99^{*}$ & $25.834^{*}$ & $95.450^{*}$ & $7.518^{*}$ & 0.769 & 0.049 \\
\hline Securities Investment Trusts & 885 & 0.116 & 3.896 & $1.001^{*}$ & $20.726^{*}$ & $15989.82^{*}$ & 13.501 & $20.863^{* *}$ & $2.067^{* *}$ & 0.687 & 0.039 \\
\hline Electricity & 885 & 0.028 & 4.528 & $-0.254^{*}$ & $9.541^{*}$ & $3366.07^{*}$ & $24.289^{*}$ & $169.419^{*}$ & $21.528^{*}$ & 0.684 & 0.020 \\
\hline Financials & 885 & 0.190 & 4.535 & 0.003 & $4.490^{*}$ & $743.554^{*}$ & 11.190 & $58.266^{*}$ & $4.519^{*}$ & 0.981 & 0.016 \\
\hline Services & 885 & $0.236^{* *}$ & 3.260 & $-0.355^{*}$ & $2.984^{*}$ & $347.041^{*}$ & $17.724^{* * * 4}$ & $55.768^{*}$ & $3.259^{*}$ & 0.852 & -0.012 \\
\hline Industrials & 885 & $0.251^{* *}$ & 3.317 & $-0.672^{*}$ & $4.793^{*}$ & $914.006^{*}$ & $19.480^{* *}$ & $108.636^{*}$ & $9.727^{*}$ & 0.918 & 0.055 \\
\hline Technology & 885 & $0.267^{* * *}$ & 4.275 & $-0.265^{*}$ & $3.677^{*}$ & $509.143^{*}$ & $19.805^{* *}$ & 8.078 & 0.938 & 0.722 & 0.001 \\
\hline Leasing \& Factoring & 885 & $0.361^{* * *}$ & 5.483 & $0.200^{* *}$ & $10.728^{*}$ & $4250.49^{*}$ & $26.666^{*}$ & $156.977^{*}$ & $12.445^{*}$ & 0.565 & -0.013 \\
\hline Wholesale \& Retail Trade & 885 & $0.310^{* *}$ & 3.641 & 0.000 & $6.697^{*}$ & $1654.24^{*}$ & $40.157^{*}$ & $122.888^{*}$ & $16.888^{*}$ & 0.676 & 0.007 \\
\hline Non-Metal Mineral Products & 885 & $0.178^{* * *}$ & 3.161 & $-0.467^{*}$ & $3.585^{*}$ & $506.332^{*}$ & $44.192^{*}$ & $49.119^{*}$ & $4.769^{*}$ & 0.791 & -0.012 \\
\hline Transportation & 885 & $0.312^{* * *}$ & 5.085 & $-0.434^{*}$ & $2.885^{*}$ & $334.874^{*}$ & 13.560 & $112.910^{*}$ & $9.417^{*}$ & 0.680 & -0.050 \\
\hline Real Estate Investment Trusts & 885 & 0.074 & 4.087 & -0.089 & $8.265^{*}$ & $2520.28^{*}$ & $30.401^{*}$ & $44.592^{*}$ & $4.612^{*}$ & 0.810 & 0.008 \\
\hline Tourism & 885 & 0.097 & 5.303 & $0.228^{*}$ & $8.307^{*}$ & $2552.71^{*}$ & $23.816^{*}$ & $22.915^{* *}$ & $2.020^{* *}$ & 0.635 & -0.052 \\
\hline
\end{tabular}

Note: *. ** and *** denote rejection of the $\mathrm{HO}$ hypothesis at the 1\%, 5\% and $10 \%$ significance levels, respectively. Q(10) and Qsq(10) are the Ljung-Box autocorrelation test statistics of order 10 for returns and squared returns respectively. ARCH(10) is the conditional heteroscedasticity test statistic of order $10 . \rho_{m i}$ denotes the unconditional correlation coefficient between the returns of sector $i$. and the BIST 100. $\rho_{\text {oi }}$ denotes the unconditional correlation coefficient between returns of sector $i$. and oil. 
Table: 2

Estimation Results of DCC-VARMA-GARCH Model for Oil and Stock Sectors

\begin{tabular}{|c|c|c|c|c|c|c|c|c|c|c|c|c|c|c|c|c|}
\hline & \multicolumn{2}{|c|}{ BIST 100} & \multicolumn{2}{|c|}{ Banks } & \multicolumn{2}{|c|}{ Information Technology } & \multicolumn{2}{|c|}{ Textile \& Leather } & \multicolumn{2}{|c|}{ Food \& Beverage } & \multicolumn{2}{|c|}{ Holding \& Investment } & \multicolumn{2}{|c|}{ Telecomm. } & \multicolumn{2}{|c|}{ Wood, Paper \& Printing } \\
\hline \multicolumn{17}{|c|}{ Conditional mean equation } \\
\hline & Stock & Oil & Stock & Oil & Stock & Oil & Stock & Oil & Stock & Oil & Stock & Oil & Stock & Oil & Stock & Oil \\
\hline$\alpha$ & $\begin{array}{l}0.279^{* *} \\
(0.117)\end{array}$ & $\begin{array}{l}0.249^{* *} \\
(0.114)\end{array}$ & $\begin{array}{l}0.293^{*} \\
(0.176)\end{array}$ & $\begin{array}{l}0.260^{* *} \\
(0.113)\end{array}$ & $\begin{array}{c}0.084 \\
(0.138) \\
\end{array}$ & $\begin{array}{c}0.213^{* * * *} \\
(0.110)\end{array}$ & $\begin{array}{l}0.268^{* *} \\
(0.113)\end{array}$ & $\begin{array}{l}0.230^{* *} \\
(0.112)\end{array}$ & \begin{tabular}{|l}
$0.292^{* *}$ \\
$(0.114)$ \\
\end{tabular} & \begin{tabular}{|l}
$0.210^{* * * *}$ \\
$(0.108)$
\end{tabular} & $\begin{array}{c}0.218^{* * * *} \\
(0.119)\end{array}$ & $\begin{array}{l}0.251^{* *} \\
(0.111)\end{array}$ & $\begin{array}{c}0.143 \\
(0.129)\end{array}$ & $\begin{array}{l}0.252^{* *} \\
(0.114)\end{array}$ & $\begin{array}{c}0.187^{* * * *} \\
(0.131)\end{array}$ & $\begin{array}{l}0.242^{* *} \\
(0.112)\end{array}$ \\
\hline$\beta$ & $\begin{array}{l}-0.029 \\
(0.034) \\
\end{array}$ & $\begin{array}{l}0.233^{*} \\
(0.033) \\
\end{array}$ & $\begin{array}{l}-0.054 \\
(0.041) \\
\end{array}$ & $\begin{array}{c}0.226^{*} \\
(0.033)\end{array}$ & $\begin{array}{l}0.080^{* *} \\
(0.035)\end{array}$ & $\begin{array}{l}0.241^{*} \\
(0.035)\end{array}$ & $\begin{array}{l}0.071^{* * *} \\
(0.040)\end{array}$ & $\begin{array}{l}0.245^{*} \\
(0.036) \\
\end{array}$ & $\begin{array}{l}-0.124^{*} \\
(0.036) \\
\end{array}$ & $\begin{array}{l}0.242^{*} \\
(0.033) \\
\end{array}$ & $\begin{array}{c}0.008 \\
(0.034)\end{array}$ & $\begin{array}{l}0.237^{*} \\
(0.036)\end{array}$ & $\begin{array}{c}-0.060^{* * * *} \\
(0.033)\end{array}$ & $\begin{array}{l}0.240^{*} \\
(0.034) \\
\end{array}$ & $\begin{array}{c}0.035 \\
(0.036) \\
\end{array}$ & $\begin{array}{l}0.251^{*} \\
(0.033)\end{array}$ \\
\hline \multicolumn{17}{|c|}{ Conditional variance equation } \\
\hline constant & $\begin{array}{c}0.226 \\
(0.140) \\
\end{array}$ & $\begin{array}{l}0.247^{* *} \\
(0.121) \\
\end{array}$ & $\begin{array}{c}0.491^{* * * *} \\
(0.258)\end{array}$ & $\begin{array}{c}0.222 \\
(0.232) \\
\end{array}$ & $\begin{array}{c}0.258 \\
(0.228) \\
\end{array}$ & $\begin{array}{l}0.296^{*} \\
(0.166) \\
\end{array}$ & $\begin{array}{c}0.875 \\
(0.775)\end{array}$ & $\begin{array}{c}0.392 \\
(0.251) \\
\end{array}$ & \begin{tabular}{|c|}
$4.515^{*}$ \\
$(0.344)$ \\
\end{tabular} & $\begin{array}{l}-3.128^{*} \\
(0.216) \\
\end{array}$ & $\begin{array}{c}0.154 \\
(0.111)\end{array}$ & $\begin{array}{l}0.239^{* * *} \\
(0.128)\end{array}$ & $\begin{array}{l}0.174^{*} \\
(0.065)\end{array}$ & \begin{tabular}{|c|}
0.178 \\
$(0.118)$ \\
\end{tabular} & $\begin{array}{c}0.669 \\
(0.559) \\
\end{array}$ & $\begin{array}{c}0.187 \\
(0.115) \\
\end{array}$ \\
\hline$\left(\varepsilon_{S, t-1}\right)^{2}$ & $\begin{array}{l}0.053^{*} \\
(0.013)\end{array}$ & $\begin{array}{l}-0.002 \\
(0.014)\end{array}$ & $\begin{array}{c}0.046^{*} \\
(0.011)\end{array}$ & $\begin{array}{l}-0.005 \\
(0.023)\end{array}$ & $\begin{array}{l}0.034^{* *} \\
(0.016)\end{array}$ & $\begin{array}{c}0.008 \\
(0.015)\end{array}$ & $\begin{array}{l}0.095^{* *} \\
(0.039)\end{array}$ & $\begin{array}{c}0.007 \\
(0.021)\end{array}$ & $\begin{array}{l}0.067^{* *} \\
(0.026)\end{array}$ & $\begin{array}{l}0.093^{* *} \\
(0.030)\end{array}$ & $\begin{array}{l}0.054^{*} \\
(0.016)\end{array}$ & $\begin{array}{c}0.004 \\
(0.012)\end{array}$ & $\begin{array}{l}0.033^{*} \\
(0.004)\end{array}$ & $\begin{array}{c}0.012 \\
(0.011)\end{array}$ & $\begin{array}{l}0.092^{* *} \\
(0.039)\end{array}$ & $\begin{array}{c}0.016 \\
(0.026)\end{array}$ \\
\hline$\left(\varepsilon_{O, t-1}\right)^{2}$ & $\begin{array}{l}0.020^{* * *} \\
(0.010)\end{array}$ & $\begin{array}{l}0.061^{*} \\
(0.013)\end{array}$ & $\begin{array}{l}0.015^{* * *} \\
(0.009)\end{array}$ & $\begin{array}{l}0.060^{*} \\
(0.019)\end{array}$ & $\begin{array}{l}0.014^{* * *} \\
(0.007)\end{array}$ & $\begin{array}{l}0.057^{*} \\
(0.017)\end{array}$ & $\begin{array}{l}0.038^{* *} \\
(0.017)\end{array}$ & $\begin{array}{l}0.060^{*} \\
(0.015)\end{array}$ & \begin{tabular}{|c|}
0.005 \\
$(0.015)$
\end{tabular} & $\begin{array}{c}0.017 \\
(0.019)\end{array}$ & $\begin{array}{l}0.013^{* * *} \\
(0.008)\end{array}$ & $\begin{array}{l}0.060^{*} \\
(0.013)\end{array}$ & $\begin{array}{l}0.016^{* * *} \\
(0.009)\end{array}$ & $\begin{array}{l}0.064^{*} \\
(0.011)\end{array}$ & $\begin{array}{l}-0.0004 \\
(0.0091)\end{array}$ & $\begin{array}{l}0.064^{*} \\
(0.014)\end{array}$ \\
\hline$h_{S, t-1}$ & $\begin{array}{l}0.923^{*} \\
(0.020)\end{array}$ & $\begin{array}{c}0.009 \\
(0.022)\end{array}$ & $\begin{array}{c}0.930^{*} \\
(0.020)\end{array}$ & $\begin{array}{c}0.013 \\
(0.039)\end{array}$ & $\begin{array}{l}0.935^{*} \\
(0.028)\end{array}$ & $\begin{array}{c}0.013 \\
(0.032)\end{array}$ & $\begin{array}{l}0.797^{*} \\
(0.095)\end{array}$ & $\begin{array}{c}0.031 \\
(0.034)\end{array}$ & $\begin{array}{l}0.227^{*} \\
(0.058)\end{array}$ & $\begin{array}{l}0.217^{*} \\
(0.052)\end{array}$ & $\begin{array}{l}0.930^{*} \\
(0.020)\end{array}$ & $\begin{array}{c}0.004 \\
(0.020)\end{array}$ & $\begin{array}{c}0.962^{*} \\
(0.004)\end{array}$ & $\begin{array}{l}-0.019 \\
(0.013)\end{array}$ & $\begin{array}{l}0.831^{*} \\
(0.082)\end{array}$ & $\begin{array}{c}0.023 \\
(0.035)\end{array}$ \\
\hline$h_{O, t-1}$ & $\begin{array}{l}-0.021 \\
(0.019)\end{array}$ & $\begin{array}{c}0.927^{*} \\
(0.015) \\
\end{array}$ & $\begin{array}{c}-0.013 \\
(0.018) \\
\end{array}$ & $\begin{array}{l}0.925^{* * * *} \\
(0.026)\end{array}$ & $\begin{array}{l}-0.024 \\
(0.022) \\
\end{array}$ & $\begin{array}{l}0.937^{*} \\
(0.027)\end{array}$ & $\begin{array}{l}-0.054 \\
(0.046)\end{array}$ & $\begin{array}{l}0.931^{*} \\
(0.027)\end{array}$ & \begin{tabular}{|c|}
$0.565^{*}$ \\
$(0.024)$ \\
\end{tabular} & $\begin{array}{c}0.702^{*} \\
(0.041)\end{array}$ & $\begin{array}{l}-0.015 \\
(0.013) \\
\end{array}$ & $\begin{array}{l}0.929^{*} \\
(0.016)\end{array}$ & $\begin{array}{l}-0.005 \\
(0.010)\end{array}$ & $\begin{array}{l}0.913^{*} \\
(0.013)\end{array}$ & $\begin{array}{c}0.004 \\
(0.018)\end{array}$ & $\begin{array}{l}0.922^{*} \\
(0.019)\end{array}$ \\
\hline$\theta_{1}$ & \multicolumn{2}{|c|}{$\begin{array}{l}0.0567^{* *} \\
(0.0257)\end{array}$} & \multicolumn{2}{|c|}{$\begin{array}{l}0.0614^{* * *} \\
(0.0359)\end{array}$} & \multicolumn{2}{|c|}{$\begin{array}{c}0.0487^{* * * *} \\
(0.0280)\end{array}$} & \multicolumn{2}{|r|}{$\begin{array}{c}0.0124 \\
(0.0189)\end{array}$} & \multicolumn{2}{|c|}{$\begin{array}{c}0.0207^{* * *} \\
(0.0112) \\
\end{array}$} & \multicolumn{2}{|c|}{$\begin{array}{c}0.0345 \\
(0.0230) \\
\end{array}$} & \multicolumn{2}{|c|}{$\begin{array}{l}0.0997^{* *} \\
(0.0490)\end{array}$} & \multicolumn{2}{|c|}{$\begin{array}{c}0.0302 \\
(0.0301) \\
\end{array}$} \\
\hline$\theta_{2}$ & \multicolumn{2}{|c|}{$\begin{array}{l}0.7684^{*} \\
(0.1006)\end{array}$} & \multicolumn{2}{|c|}{$\begin{array}{l}0.6794^{*} \\
(0.1611)\end{array}$} & \multicolumn{2}{|c|}{$\begin{array}{l}0.7432^{*} \\
(0.1661)\end{array}$} & \multicolumn{2}{|c|}{$\begin{array}{l}0.8504^{*} \\
(0.2099)\end{array}$} & \multicolumn{2}{|c|}{$\begin{array}{c}0.9558^{*} \\
(0.0162)\end{array}$} & \multicolumn{2}{|c|}{$\begin{array}{c}0.7954^{*} \\
(0.1575)\end{array}$} & \multicolumn{2}{|c|}{$\begin{array}{c}0.0578 \\
(0.2802)\end{array}$} & \multicolumn{2}{|c|}{$\begin{array}{c}0.1509^{*} \\
(0.5284)\end{array}$} \\
\hline$Q(10)$ & $\begin{array}{l}10.553 \\
{[0.393]}\end{array}$ & \begin{tabular}{|l|}
13.878 \\
{$[0.179]$} \\
\end{tabular} & $\begin{array}{c}7.562 \\
{[0.672]}\end{array}$ & $\begin{array}{l}13.888 \\
{[0.178]}\end{array}$ & $\begin{array}{l}15.211 \\
{[0.125]}\end{array}$ & $\begin{array}{l}13.731 \\
{[0.186]}\end{array}$ & $\begin{array}{l}12.051 \\
{[0.282]}\end{array}$ & $\begin{array}{l}13.408 \\
{[0.202]}\end{array}$ & \begin{tabular}{|c|}
10.754 \\
{$[0.377]$} \\
\end{tabular} & \begin{tabular}{|l|}
14.011 \\
{$[0.172]$}
\end{tabular} & $\begin{array}{l}10.711 \\
{[0.380]}\end{array}$ & $\begin{array}{l}13.735 \\
{[0.185]}\end{array}$ & $\begin{array}{c}9.974 \\
{[0.443]}\end{array}$ & \begin{tabular}{|l|}
13.972 \\
{$[0.082]$} \\
\end{tabular} & $\begin{array}{l}10.747 \\
{[0.377]}\end{array}$ & $\begin{array}{l}13.740 \\
{[0.185]}\end{array}$ \\
\hline$Q^{2}(10)$ & $\begin{array}{c}5.787 \\
{[0.671]}\end{array}$ & $\begin{array}{c}7.401 \\
{[0.494]}\end{array}$ & $\begin{array}{c}3.631 \\
{[0.889]}\end{array}$ & $\begin{array}{c}8.303 \\
{[0.404]}\end{array}$ & $\begin{array}{c}9.336 \\
{[0.315]}\end{array}$ & $\begin{array}{c}6.509 \\
{[0.590]}\end{array}$ & $\begin{array}{c}4.467 \\
{[0.813]}\end{array}$ & $\begin{array}{l}10.468 \\
{[0.234]}\end{array}$ & $\begin{array}{c}8.643 \\
{[0.373]}\end{array}$ & $\begin{array}{c}7.373 \\
{[0.497]}\end{array}$ & $\begin{array}{c}6.480 \\
{[0.594]}\end{array}$ & $\begin{array}{c}6.736 \\
{[0.565]}\end{array}$ & $\begin{array}{l}14.857 \\
{[0.137]}\end{array}$ & $\begin{array}{c}9.038 \\
{[0.339]}\end{array}$ & $\begin{array}{c}5.528 \\
{[0.700]}\end{array}$ & $\begin{array}{c}6.000 \\
{[0.647]}\end{array}$ \\
\hline $\mathrm{ARCH}(10)$ & $\begin{array}{c}0.558 \\
{[0.849]}\end{array}$ & $\begin{array}{c}0.770 \\
{[0.658]}\end{array}$ & $\begin{array}{c}0.293 \\
{[0.983]}\end{array}$ & $\begin{array}{c}0.868 \\
{[0.563]}\end{array}$ & $\begin{array}{c}1.056 \\
{[0.394]}\end{array}$ & $\begin{array}{c}0.688 \\
{[0.736]}\end{array}$ & $\begin{array}{c}0.672 \\
{[0.751]}\end{array}$ & $\begin{array}{c}1.021 \\
{[0.424]}\end{array}$ & $\begin{array}{c}0.850 \\
{[0.581]} \\
\end{array}$ & $\begin{array}{c}0.742 \\
{[0.685]}\end{array}$ & $\begin{array}{c}0.684 \\
{[0.740]}\end{array}$ & $\begin{array}{c}0.711 \\
{[0.714]}\end{array}$ & $\begin{array}{c}0.782 \\
{[0.646]}\end{array}$ & $\begin{array}{c}0.919 \\
{[0.515]}\end{array}$ & $\begin{array}{c}0.532 \\
{[0.868]}\end{array}$ & $\begin{array}{c}0.658 / \\
{[0.764]}\end{array}$ \\
\hline$J B$ & $\begin{array}{l}167.574 \\
{[0.000]}\end{array}$ & $\begin{array}{l}93.077 \\
{[0.000]}\end{array}$ & $\begin{array}{c}317.812 \\
{[0.000]} \\
\end{array}$ & $\begin{array}{l}23.591 \\
{[0.000]}\end{array}$ & $\begin{array}{c}465.846 \\
{[0.000]} \\
\end{array}$ & $\begin{array}{l}79.774 \\
{[0.000]}\end{array}$ & $\begin{array}{l}62.526 \\
{[0.000]} \\
\end{array}$ & $409.219[0.000]$ & \begin{tabular}{|l|}
45.537 \\
{$[0.000]$} \\
\end{tabular} & $\begin{array}{l}29.214 \\
{[0.000]}\end{array}$ & $\begin{array}{l}156.700 \\
{[0.000]} \\
\end{array}$ & $\begin{array}{l}80.300 \\
{[0.000]}\end{array}$ & $\begin{array}{l}54.749 \\
{[0.000]}\end{array}$ & $\begin{array}{l}30.331 \\
{[0.000]}\end{array}$ & $\begin{array}{l}42.492 \\
{[0.000]}\end{array}$ & $\begin{array}{l}83.896 \\
{[0.000]}\end{array}$ \\
\hline$\rho_{S O}^{m}$ & \multicolumn{2}{|c|}{0.0165} & \multicolumn{2}{|c|}{-0.0047} & \multicolumn{2}{|c|}{-0.0352} & \multicolumn{2}{|r|}{0.0113} & \multicolumn{2}{|c|}{0.0038} & & & -0.0 & & & \\
\hline AIC & 10. & & 11. & & & & & 10.904 & 10. & 910 & & & 11. & & & \\
\hline
\end{tabular}


Table: 2

Estimation Results of DCC-VARMA-GARCH Model for Oil and Stock Sectors (Continued)

\begin{tabular}{|c|c|c|c|c|c|c|c|c|c|c|c|c|c|c|c|c|}
\hline & \multicolumn{2}{|c|}{ Chemical, Petrol \& Plastic } & \multicolumn{2}{|c|}{ Basic Metal } & \multicolumn{2}{|c|}{ Metal Products \& Machinery } & \multicolumn{2}{|c|}{ Insurance } & \multicolumn{2}{|c|}{ Securities Investment Trusts } & \multicolumn{2}{|c|}{ Electricity } & \multicolumn{2}{|c|}{ Financials } & \multicolumn{2}{|c|}{ Services } \\
\hline \multicolumn{17}{|c|}{ Conditional mean equation } \\
\hline & Stock & Oil & Stock & Oil & Stock & Oil & Stock & Oil & Stock & Oil & Stock & Oil & Stock & Oil & Stock & Oil \\
\hline$\alpha$ & $\begin{array}{l}0.381^{*} \\
(0.120)\end{array}$ & $\begin{array}{l}0.248^{* *} \\
(0.111)\end{array}$ & \begin{tabular}{|l}
$0.496^{*}$ \\
$(0.144)$ \\
\end{tabular} & $\begin{array}{l}0.273^{* *} \\
(0.113) \\
\end{array}$ & $\begin{array}{l}0.260^{* *} \\
(0.115)\end{array}$ & $\begin{array}{l}0.236^{* *} \\
(0.116)\end{array}$ & $\begin{array}{l}0.270^{*} \\
(0.102)\end{array}$ & $\begin{array}{l}0.254^{* *} \\
(0.113)\end{array}$ & $\begin{array}{c}0.060 \\
(0.126)\end{array}$ & $\begin{array}{l}0.246^{* *} \\
(0.106)\end{array}$ & $\begin{array}{c}0.036 \\
(0.136)\end{array}$ & $\begin{array}{l}0.241^{* *} \\
(0.113)\end{array}$ & $\begin{array}{c}0.248^{* * * *} \\
(0.135)\end{array}$ & $\begin{array}{l}0.253^{* *} \\
(0.115) \\
\end{array}$ & $\begin{array}{l}0.288^{*} \\
(0.100)\end{array}$ & $\begin{array}{l}0.242^{* *} \\
(0.118)\end{array}$ \\
\hline$\beta$ & $\begin{array}{l}-0.054 \\
(0.036) \\
\end{array}$ & $\begin{array}{l}0.230^{*} \\
(0.033) \\
\end{array}$ & $\begin{array}{c}0.009 \\
(0.034) \\
\end{array}$ & $\begin{array}{l}0.237^{*} \\
(0.035) \\
\end{array}$ & $\begin{array}{c}0.009 \\
(0.035) \\
\end{array}$ & $\begin{array}{l}0.237^{*} \\
(0.037)\end{array}$ & $\begin{array}{l}0.058^{* * * *} \\
(0.034) \\
\end{array}$ & $\begin{array}{l}0.239^{*} \\
(0.035)\end{array}$ & $\begin{array}{c}0.047 \\
(0.041) \\
\end{array}$ & $\begin{array}{l}0.237^{*} \\
(0.028) \\
\end{array}$ & $\begin{array}{c}0.019 \\
(0.043) \\
\end{array}$ & $\begin{array}{l}0.243^{*} \\
(0.035) \\
\end{array}$ & $\begin{array}{l}-0.030 \\
(0.033) \\
\end{array}$ & $\begin{array}{l}0.231^{*} \\
(0.033)\end{array}$ & $\begin{array}{l}-0.030 \\
(0.035) \\
\end{array}$ & $\begin{array}{l}0.238^{*} \\
(0.035) \\
\end{array}$ \\
\hline \multicolumn{17}{|c|}{ Conditional variance equation } \\
\hline constant & $\begin{array}{l}0.582^{* *} \\
(0.244) \\
\end{array}$ & $\begin{array}{l}0.258^{* * *} \\
(0.137) \\
\end{array}$ & \begin{tabular}{|c|}
0.968 \\
$(0.757)$ \\
\end{tabular} & \begin{tabular}{|l|}
-0.157 \\
$(0.202)$ \\
\end{tabular} & $\begin{array}{l}0.328^{* *} \\
(0.138)\end{array}$ & $\begin{array}{l}0.216^{* *} \\
(0.104)\end{array}$ & $\begin{array}{c}0.070 \\
(0.077) \\
\end{array}$ & $\begin{array}{l}0.222^{* *} \\
(0.106) \\
\end{array}$ & $\begin{array}{c}0.063 \\
(0.074) \\
\end{array}$ & $\begin{array}{c}0.195 \\
(0.137)\end{array}$ & $\begin{array}{l}3.174^{* *} \\
(1.463)\end{array}$ & $\begin{array}{c}0.430 \\
(0.298) \\
\end{array}$ & $\begin{array}{l}0.288^{* * * *} \\
(0.200)\end{array}$ & $\begin{array}{c}0.250 \\
(0.177) \\
\end{array}$ & $\begin{array}{c}0.301 \\
(0.200) \\
\end{array}$ & $\begin{array}{l}0.264^{* * 4} \\
(0.139)\end{array}$ \\
\hline$\left(\varepsilon_{S, t-1}\right)^{2}$ & $\begin{array}{l}0.066^{*} \\
(0.018)\end{array}$ & $\begin{array}{l}-0.002 \\
(0.013)\end{array}$ & $\begin{array}{l}0.084^{*} \\
(0.027)\end{array}$ & $\begin{array}{c}0.027 \\
(0.024)\end{array}$ & $\begin{array}{l}0.076^{*} \\
(0.013)\end{array}$ & $\begin{array}{c}0.008 \\
(0.009)\end{array}$ & $\begin{array}{c}0.045 \\
(0.030)\end{array}$ & $\begin{array}{c}0.003 \\
(0.004)\end{array}$ & $\begin{array}{l}0.025^{* * *} \\
(0.013)\end{array}$ & $\begin{array}{c}0.009 \\
(0.009)\end{array}$ & $\begin{array}{l}0.153^{*} \\
(0.051)\end{array}$ & $\begin{array}{c}0.013 \\
(0.036)\end{array}$ & $\begin{array}{l}0.049^{*} \\
(0.014)\end{array}$ & $\begin{array}{l}-0.005 \\
(0.022)\end{array}$ & $\begin{array}{l}0.057^{* *} \\
(0.024)\end{array}$ & $\begin{array}{c}0.008 \\
(0.007)\end{array}$ \\
\hline$\left(\varepsilon_{O, t-1}\right)^{2}$ & $\begin{array}{l}0.015^{* *} \\
(0.007)\end{array}$ & $\begin{array}{l}0.064^{*} \\
(0.013)\end{array}$ & $\begin{array}{c}0.002 \\
(0.008)\end{array}$ & $\begin{array}{l}0.051^{*} \\
(0.016)\end{array}$ & $\begin{array}{l}0.010^{* * *} \\
(0.006)\end{array}$ & $\begin{array}{l}0.066^{*} \\
(0.012)\end{array}$ & $\begin{array}{c}0.008 \\
(0.006)\end{array}$ & $\begin{array}{l}0.060^{*} \\
(0.014)\end{array}$ & $\begin{array}{c}0.005 \\
(0.006)\end{array}$ & $\begin{array}{l}0.069^{*} \\
(0.014)\end{array}$ & $\begin{array}{c}0.015 \\
(0.011)\end{array}$ & $\begin{array}{l}0.062^{*} \\
(0.014)\end{array}$ & $\begin{array}{l}0.016^{* * *} \\
(0.009)\end{array}$ & $\begin{array}{l}0.059^{*} \\
(0.016)\end{array}$ & $\begin{array}{l}0.024^{* * *} \\
(0.013)\end{array}$ & $\begin{array}{l}0.065^{*} \\
(0.014)\end{array}$ \\
\hline$h_{S, t-1}$ & $\begin{array}{c}0.890^{*} \\
(0.029)\end{array}$ & $\begin{array}{c}0.004 \\
(0.022)\end{array}$ & $\begin{array}{c}0.826^{*} \\
(0.109)\end{array}$ & $\begin{array}{c}0.038 \\
(0.074)\end{array}$ & $\begin{array}{c}0.894^{*} \\
(0.021)\end{array}$ & $\begin{array}{c}0.002 \\
(0.012)\end{array}$ & $\begin{array}{l}0.954^{*} \\
(0.028)\end{array}$ & $\begin{array}{l}-0.006 \\
(0.007)\end{array}$ & $\begin{array}{l}0.971^{*} \\
(0.010)\end{array}$ & $\begin{array}{l}-0.009 \\
(0.010)\end{array}$ & $\begin{array}{c}0.643^{*} \\
(0.085)\end{array}$ & $\begin{array}{c}0.044 \\
(0.055)\end{array}$ & $\begin{array}{c}0.930^{*} \\
(0.020)\end{array}$ & $\begin{array}{c}0.014 \\
(0.036)\end{array}$ & $\begin{array}{c}0.909^{*} \\
(0.039)\end{array}$ & $\begin{array}{l}-0.005 \\
(0.009)\end{array}$ \\
\hline$h_{O, t-1}$ & $\begin{array}{c}-0.017^{* * *} \\
(0.009)\end{array}$ & $\begin{array}{l}0.923^{*} \\
(0.014)\end{array}$ & $\begin{array}{l}0.051^{*} \\
(0.008)\end{array}$ & $\begin{array}{l}0.887^{*} \\
(0.018) \\
\end{array}$ & $\begin{array}{l}-0.013 \\
(0.009) \\
\end{array}$ & $\begin{array}{l}0.926^{*} \\
(0.013)\end{array}$ & $\begin{array}{c}-0.007 \\
(0.009) \\
\end{array}$ & $\begin{array}{c}0.926^{*} \\
(0.015)\end{array}$ & $\begin{array}{c}0.004 \\
(0.010)\end{array}$ & $\begin{array}{c}0.914^{*} \\
(0.017)\end{array}$ & $\begin{array}{c}-0.030 \\
(0.029) \\
\end{array}$ & $\begin{array}{c}0.932^{*} \\
(0.020)\end{array}$ & $\begin{array}{c}-0.017 \\
(0.016)\end{array}$ & $\begin{array}{c}0.928^{*} \\
(0.019)\end{array}$ & $\begin{array}{l}-0.026 \\
(0.024)\end{array}$ & $\begin{array}{c}0.921^{*} \\
(0.017) \\
\end{array}$ \\
\hline$\theta_{1}$ & \multicolumn{2}{|c|}{$\begin{array}{c}1 \\
0.0590^{*} \\
(0.0173) \\
\end{array}$} & \multicolumn{2}{|c|}{$\begin{array}{l}0.0869^{*} \\
(0.0304) \\
\end{array}$} & \multicolumn{2}{|c|}{$\begin{array}{c}0.0667^{*} \\
(0.0249) \\
\end{array}$} & \multicolumn{2}{|c|}{$\begin{array}{c}0.0372^{* * *} \\
(0.0216)\end{array}$} & \multicolumn{2}{|c|}{$\begin{array}{c}0.0336 \\
(0.0214) \\
\end{array}$} & \multicolumn{2}{|c|}{$\begin{array}{l}0.0462^{* *} \\
(0.0226)\end{array}$} & \multicolumn{2}{|c|}{$\begin{array}{l}0.0505^{* * *} \\
(0.0298)\end{array}$} & \multicolumn{2}{|c|}{$\begin{array}{l}0.1193^{*} \\
(0.0455) \\
\end{array}$} \\
\hline$\theta_{2}$ & \multicolumn{2}{|c|}{$\begin{array}{c}0.8838^{*} \\
(0.0337)\end{array}$} & \multicolumn{2}{|c|}{$\begin{array}{l}0.6980^{*} \\
(0.1023)\end{array}$} & \multicolumn{2}{|c|}{$\begin{array}{c}0.8205^{*} \\
(0.0920)\end{array}$} & \multicolumn{2}{|c|}{$\begin{array}{l}0.8108^{*} \\
(0.1231)\end{array}$} & \multicolumn{2}{|c|}{$\begin{array}{l}0.8926^{*} \\
(0.1081)\end{array}$} & \multicolumn{2}{|c|}{$\begin{array}{l}0.8303^{*} \\
(0.0751)\end{array}$} & \multicolumn{2}{|c|}{$\begin{array}{l}0.7292^{*} \\
(0.1483)\end{array}$} & \multicolumn{2}{|c|}{$\begin{array}{c}0.1311 \\
(0.1882)\end{array}$} \\
\hline$Q(10)$ & $\begin{array}{c}9.892 \\
{[0.450]}\end{array}$ & $\begin{array}{l}14.072 \\
{[0.170]}\end{array}$ & $\begin{array}{l}15.911 \\
{[0.102]}\end{array}$ & $\begin{array}{l}13.331 \\
{[0.206]}\end{array}$ & $\begin{array}{l}13.596 \\
{[0.192]}\end{array}$ & $\begin{array}{l}13.980 \\
{[0.174]}\end{array}$ & $\begin{array}{l}15.114 \\
{[0.128]}\end{array}$ & $\begin{array}{l}13.517 \\
{[0.196]}\end{array}$ & $\begin{array}{c}2.991 \\
{[0.982]}\end{array}$ & $\begin{array}{l}14.105 \\
{[0.168]}\end{array}$ & $\begin{array}{l}12.281 \\
{[0.267]}\end{array}$ & $\begin{array}{l}13.463 \\
{[0.199]}\end{array}$ & $\begin{array}{c}7.467 \\
{[0.681]}\end{array}$ & \begin{tabular}{|c|}
13.813 \\
{$[0.182]$}
\end{tabular} & $\begin{array}{l}12.082 \\
{[0.280]}\end{array}$ & \begin{tabular}{|c|}
14.083 \\
{$[0.169]$}
\end{tabular} \\
\hline$Q^{2}(10)$ & $\begin{array}{c}6.910 \\
{[0.546]}\end{array}$ & $\begin{array}{c}7.553 \\
{[0.478]}\end{array}$ & $\begin{array}{c}7.620 \\
{[0.471]}\end{array}$ & $\begin{array}{c}9.706 \\
{[0.286]}\end{array}$ & $\begin{array}{c}6.585 \\
{[0.582]}\end{array}$ & $\begin{array}{c}6.170 \\
{[0.628]}\end{array}$ & $\begin{array}{c}7.843 \\
{[0.449]}\end{array}$ & $\begin{array}{c}6.602 \\
{[0.580]}\end{array}$ & $\begin{array}{c}1.807 \\
{[0.986]}\end{array}$ & $\begin{array}{c}7.512 \\
{[0.483]}\end{array}$ & $\begin{array}{c}9.428 \\
{[0.307]}\end{array}$ & $\begin{array}{c}6.750 \\
{[0.564]}\end{array}$ & $\begin{array}{c}3.624 \\
{[0.889]}\end{array}$ & $\begin{array}{c}7.758 \\
{[0.457]}\end{array}$ & $\begin{array}{c}9.196 \\
{[0.326]}\end{array}$ & $\begin{array}{c}7.150 \\
{[0.521]}\end{array}$ \\
\hline $\mathrm{ARCH}(10)$ & $\begin{array}{c}0.901 \\
{[0.531]}\end{array}$ & $\begin{array}{c}0.782 \\
{[0.647]}\end{array}$ & \begin{tabular}{|c|}
0.673 \\
{$[0.751]$}
\end{tabular} & $\begin{array}{c}1.013 \\
{[0.430]}\end{array}$ & $\begin{array}{c}0.713 \\
{[0.712]}\end{array}$ & $\begin{array}{c}0.659 \\
{[0.763}\end{array}$ & $\begin{array}{c}0.807 \\
{[0.622]}\end{array}$ & $\begin{array}{c}0.712 \\
{[0.714]}\end{array}$ & $\begin{array}{c}0.178 \\
{[0.998]}\end{array}$ & $\begin{array}{c}0.787 \\
{[0.642]}\end{array}$ & $\begin{array}{c}0.859 \\
{[0.571]}\end{array}$ & $\begin{array}{c}0.706 \\
{[0.719]}\end{array}$ & $\begin{array}{c}0.297 \\
{[0.982]}\end{array}$ & $\begin{array}{c}0.810 \\
{[0.619]}\end{array}$ & $\begin{array}{c}0.663 \\
{[0.760]}\end{array}$ & $\begin{array}{c}0.732 \\
{[0.694]}\end{array}$ \\
\hline$J B$ & $\begin{array}{l}196.370 \\
{[0.000]} \\
\end{array}$ & $\begin{array}{l}117.888 \\
{[0.000]} \\
\end{array}$ & $\begin{array}{l}66.155 \\
{[0.000]} \\
\end{array}$ & $\begin{array}{l}27.943 \\
{[0.000]}\end{array}$ & $\begin{array}{l}98.756 \\
{[0.000]}\end{array}$ & $\begin{array}{l}96.101 \\
{[0.000]}\end{array}$ & $\begin{array}{l}166.339 \\
{[0.000]}\end{array}$ & $\begin{array}{l}155.054 \\
{[0.000]}\end{array}$ & $\begin{array}{l}4886.03 \\
{[0.000]} \\
\end{array}$ & $\begin{array}{l}301.09 \\
{[0.000]}\end{array}$ & $\begin{array}{l}246.900 \\
{[0.000]} \\
\end{array}$ & $\begin{array}{l}114.903 \\
{[0.000]}\end{array}$ & $\begin{array}{l}234.084 \\
{[0.000]} \\
\end{array}$ & $\begin{array}{l}79.637 \\
{[0.000]}\end{array}$ & $\begin{array}{l}59.510 \\
{[0.000]}\end{array}$ & $\begin{array}{l}150.181 \\
{[0.000]}\end{array}$ \\
\hline$\rho_{S O}^{m}$ & \multicolumn{2}{|c|}{0.0663} & \multicolumn{2}{|c|}{0.0513} & \multicolumn{2}{|c|}{0.0167} & \multicolumn{2}{|c|}{0.0414} & \multicolumn{2}{|c|}{0.0304} & 0.0 & & 0.0 & & & \\
\hline AIC & & & 11 & 353 & & & 10. & & & & 11. & & 11. & & & 647 \\
\hline
\end{tabular}


Table: 2

Estimation Results of DCC-VARMA-GARCH Model for Oil and Stock Sectors (Continued)

\begin{tabular}{|c|c|c|c|c|c|c|c|c|c|c|c|c|c|c|c|c|}
\hline \multicolumn{3}{|c|}{\begin{tabular}{l|r} 
& Industrials \\
\end{tabular}} & \multicolumn{2}{|c|}{ Technology } & \multicolumn{2}{|c|}{ Leasing \& Factoring } & \multicolumn{2}{|c|}{ Wholesale \& Retail Trade } & \multicolumn{2}{|c|}{ Non-Metal Mineral Products } & \multicolumn{2}{|c|}{ Transportation } & \multicolumn{2}{|c|}{ REIT } & \multicolumn{2}{|c|}{ Tourism } \\
\hline \\
\hline & Stock & Oil & Stock & Oil & Stock & Oil & Stock & Oil & Stock & Oil & Stock & Oil & Stock & Oil & Stock & Oil \\
\hline$\alpha$ & $\begin{array}{l}0.323^{*} \\
(0.096)\end{array}$ & $\begin{array}{l}0.238^{* *} \\
(0.114)\end{array}$ & $\begin{array}{l}0.316^{* *} \\
(0.157)\end{array}$ & $\begin{array}{l}0.231^{* *} \\
(0.114)\end{array}$ & $\begin{array}{c}0.179 \\
(0.131)\end{array}$ & $\begin{array}{l}0.240^{* *} \\
(0.112)\end{array}$ & $\begin{array}{l}0.274^{* *} \\
(0.116)\end{array}$ & $\begin{array}{l}0.239^{* *} \\
(0.113)\end{array}$ & $\begin{array}{c}0.097 \\
(0.101)\end{array}$ & $\begin{array}{l}0.243^{* *} \\
(0.113)\end{array}$ & $\begin{array}{c}0.221 \\
(0.161)\end{array}$ & $\begin{array}{l}0.230^{* *} \\
(0.114)\end{array}$ & $\begin{array}{c}0.005 \\
(0.136)\end{array}$ & $\begin{array}{l}0.263^{* *} \\
(0.115)\end{array}$ & $\begin{array}{c}0.004 \\
(0.141)\end{array}$ & $\begin{array}{l}0.267^{* *} \\
(0.112)\end{array}$ \\
\hline$\beta_{1}$ & $\begin{array}{l}-0.014 \\
(0.039) \\
\end{array}$ & $\begin{array}{l}0.235^{*} \\
(0.035)\end{array}$ & $\begin{array}{c}0.043 \\
(0.036) \\
\end{array}$ & $\begin{array}{l}0.244^{*} \\
(0.032) \\
\end{array}$ & $\begin{array}{c}0.048 \\
(0.031)\end{array}$ & $\begin{array}{l}0.240^{*} \\
(0.034) \\
\end{array}$ & $\begin{array}{l}-0.048 \\
(0.039)\end{array}$ & $\begin{array}{l}0.262^{*} \\
(0.034)\end{array}$ & $\begin{array}{l}0.095^{*} \\
(0.031)\end{array}$ & $\begin{array}{l}0.260^{*} \\
(0.037)\end{array}$ & $\begin{array}{c}0.047 \\
(0.036) \\
\end{array}$ & $\begin{array}{l}0.260^{*} \\
(0.032) \\
\end{array}$ & $\begin{array}{c}0.054 \\
(0.042) \\
\end{array}$ & $\begin{array}{l}0.260^{*} \\
(0.035)\end{array}$ & $\begin{array}{l}0.067^{* * * *} \\
(0.035) \\
\end{array}$ & $\begin{array}{l}0.263^{*} \\
(0.037)\end{array}$ \\
\hline$\beta_{2}$ & & & & & & & $\begin{array}{l}0.081^{* *} \\
(0.034)\end{array}$ & $\begin{array}{l}-0.079^{* *} \\
(0.035)\end{array}$ & $\begin{array}{l}0.108^{*} \\
(0.030)\end{array}$ & $\begin{array}{c}-0.066^{* * *} \\
(0.035)\end{array}$ & $\begin{array}{l}0.119^{*} \\
(0.037)\end{array}$ & $\begin{array}{c}-0.065^{* * *} \\
(0.035)\end{array}$ & $\begin{array}{l}0.100^{* * *} \\
(0.043)\end{array}$ & $\begin{array}{c}-0.062^{* * * *} \\
(0.034)\end{array}$ & $\begin{array}{l}0.121^{*} \\
(0.038)\end{array}$ & $\begin{array}{l}-0.075^{* *} \\
(0.033)\end{array}$ \\
\hline$\beta_{3}$ & & & & & & & $\begin{array}{l}0.088^{*} \\
(0.031)\end{array}$ & $\begin{array}{l}0.066^{* * * *} \\
(0.037)\end{array}$ & & & & & & & & \\
\hline$d_{1}$ & & & & & & & $\begin{array}{l}-22.584^{*} \\
(0.557)\end{array}$ & & & & & & & & & \\
\hline$d_{2}$ & & & & & & & $\begin{array}{l}26.928^{*} \\
(0.994)\end{array}$ & & & & & & & & & \\
\hline \multicolumn{17}{|c|}{$\frac{1}{\text { Conditional variance equation }}$} \\
\hline constant & $\begin{array}{l}0.290^{* *} \\
(0.143) \\
\end{array}$ & $\begin{array}{l}0.265^{* * *} \\
(0.154)\end{array}$ & $\begin{array}{c}0.308 \\
(0.227) \\
\end{array}$ & $\begin{array}{c}0.244 \\
(0.244) \\
\end{array}$ & $\begin{array}{l}-0.041 \\
(0.062)\end{array}$ & $\begin{array}{c}0.188 \\
(0.116) \\
\end{array}$ & $\begin{array}{c}0.201 \\
(0.161)\end{array}$ & $\begin{array}{c}0.195 \\
(0.378)\end{array}$ & $\begin{array}{c}0.218 \\
(0.206)\end{array}$ & $\begin{array}{c}0.262 \\
(0.216)\end{array}$ & $\begin{array}{l}1.247^{* * *} \\
(0.647)\end{array}$ & $\begin{array}{c}0.518 \\
(0.335)\end{array}$ & $\begin{array}{c}0.076 \\
(0.175)\end{array}$ & $\begin{array}{c}0.244 \\
(0.174) \\
\end{array}$ & $\begin{array}{l}3.378^{* * * *} \\
(1.833)\end{array}$ & $\begin{array}{c}0.202 \\
(0.224) \\
\end{array}$ \\
\hline$\left(\varepsilon_{S, t-1}\right)^{2}$ & $\begin{array}{l}0.064^{*} \\
(0.012) \\
\end{array}$ & $\begin{array}{c}0.002 \\
(0.009) \\
\end{array}$ & $\begin{array}{l}0.034^{* *} \\
(0.017)\end{array}$ & $\begin{array}{c}0.004 \\
(0.018) \\
\end{array}$ & $\begin{array}{l}0.020^{*} \\
(0.005)\end{array}$ & $\begin{array}{c}0.007 \\
(0.010)\end{array}$ & $\begin{array}{l}0.019^{* *} \\
(0.009)\end{array}$ & $\begin{array}{c}0.004 \\
(0.007)\end{array}$ & $\begin{array}{l}0.047^{*} \\
(0.018)\end{array}$ & $\begin{array}{c}0.001 \\
(0.011)\end{array}$ & $\begin{array}{l}0.053^{*} \\
(0.017)\end{array}$ & $\begin{array}{c}0.017 \\
(0.027)\end{array}$ & $\begin{array}{c}0.014 \\
(0.019)\end{array}$ & $\begin{array}{c}0.010 \\
(0.013)\end{array}$ & $\begin{array}{l}0.260^{*} \\
(0.083)\end{array}$ & $\begin{array}{c}0.029 \\
(0.059) \\
\end{array}$ \\
\hline$\left(\varepsilon_{O, t-1}\right)^{2}$ & $\begin{array}{l}0.026^{* * * *} \\
(0.016)\end{array}$ & $\begin{array}{l}0.062^{*} \\
(0.017)\end{array}$ & $\begin{array}{c}0.010 \\
(0.009)\end{array}$ & $\begin{array}{l}0.064^{*} \\
(0.017)\end{array}$ & $\begin{array}{c}0.005 \\
(0.004)\end{array}$ & $\begin{array}{l}0.061^{*} \\
(0.016)\end{array}$ & $\begin{array}{l}-0.007 \\
(0.011)\end{array}$ & $\begin{array}{l}0.064^{3} \\
(0.015)\end{array}$ & $\begin{array}{c}0.014 \\
(0.018)\end{array}$ & $\begin{array}{l}0.062^{3} \\
(0.019)\end{array}$ & $\begin{array}{c}0.011 \\
(0.011)\end{array}$ & $\begin{array}{l}0.058^{* *} \\
(0.027)\end{array}$ & $\begin{array}{c}0.009 \\
(0.008)\end{array}$ & $\begin{array}{l}0.064^{*} \\
(0.016)\end{array}$ & $\begin{array}{c}0.008 \\
(0.012)\end{array}$ & $\begin{array}{l}0.059^{*} \\
(0.019)\end{array}$ \\
\hline$h_{S, t-1}$ & $\begin{array}{l}0.887^{*} \\
(0.008) \\
\end{array}$ & $\begin{array}{c}0.014 \\
(0.016) \\
\end{array}$ & $\begin{array}{l}0.940^{*} \\
(0.026) \\
\end{array}$ & $\begin{array}{c}0.006 \\
(0.027) \\
\end{array}$ & $\begin{array}{l}0.977^{*} \\
(0.007)\end{array}$ & $\begin{array}{l}-0.001 \\
(0.014) \\
\end{array}$ & $\begin{array}{l}0.958^{*} \\
(0.019)\end{array}$ & $\begin{array}{l}-0.002 \\
(0.009)\end{array}$ & $\begin{array}{l}0.915^{*} \\
(0.027)\end{array}$ & $\begin{array}{c}0.008 \\
(0.015) \\
\end{array}$ & $\begin{array}{l}0.887^{*} \\
(0.033)\end{array}$ & $\begin{array}{l}-0.001 \\
(0.033)\end{array}$ & $\begin{array}{c}0.979^{*} \\
(0.031)\end{array}$ & $\begin{array}{l}-0.008 \\
(0.017)\end{array}$ & $\begin{array}{l}0.559^{*} \\
(0.176)\end{array}$ & $\begin{array}{c}0.134 \\
(0.263) \\
\end{array}$ \\
\hline$h_{O, t-1}$ & $\begin{array}{l}-0.031 \\
(0.039)\end{array}$ & $\begin{array}{l}0.927^{*} \\
(0.026)\end{array}$ & $\begin{array}{l}-0.009 \\
(0.022)\end{array}$ & $\begin{array}{l}0.922^{*} \\
(0.021)\end{array}$ & $\begin{array}{l}0.0005 \\
(0.005)\end{array}$ & $\begin{array}{l}0.918^{*} \\
(0.019)\end{array}$ & $\begin{array}{c}0.008 \\
(0.042)\end{array}$ & $\begin{array}{l}0.925^{*} \\
(0.016)\end{array}$ & $\begin{array}{l}-0.027 \\
(0.043)\end{array}$ & $\begin{array}{c}0.931^{*} \\
(0.024)\end{array}$ & $\begin{array}{l}-0.027 \\
(0.026)\end{array}$ & $\begin{array}{c}0.936^{*} \\
(0.031)\end{array}$ & $\begin{array}{l}-0.013 \\
(0.015)\end{array}$ & $\begin{array}{l}0.926^{*} \\
(0.019)\end{array}$ & $\begin{array}{l}-0.005 \\
(0.024)\end{array}$ & $\begin{array}{l}0.925^{*} \\
(0.029)\end{array}$ \\
\hline$\theta_{1}$ & \multicolumn{2}{|c|}{$\begin{array}{l}0.0751^{* *} \\
(0.0320)\end{array}$} & \multicolumn{2}{|c|}{$\begin{array}{c}0.0566^{*} \\
(0.0338)\end{array}$} & \multicolumn{2}{|c|}{$\begin{array}{c}0.0118 \\
(0.0088)\end{array}$} & \multicolumn{2}{|c|}{$\begin{array}{c}0.0401 \\
(0.0379)\end{array}$} & \multicolumn{2}{|c|}{$\begin{array}{l}0.0414^{* * * *} \\
(0.0233)\end{array}$} & \multicolumn{2}{|c|}{$\begin{array}{c}0.0378^{* * *} \\
(0.0222)\end{array}$} & \multicolumn{2}{|c|}{$\begin{array}{c}0.0251 \\
(0.0214)\end{array}$} & \multicolumn{2}{|c|}{$\begin{array}{c}0.0265^{*} \\
(0.0094)\end{array}$} \\
\hline$\theta_{2}$ & \multicolumn{2}{|c|}{$\begin{array}{l}0.7884^{*} \\
(0.1184)\end{array}$} & \multicolumn{2}{|c|}{$\begin{array}{c}0.2639 \\
(0.3225)\end{array}$} & \multicolumn{2}{|c|}{$\begin{array}{l}0.9635^{*} \\
(0.0336)\end{array}$} & \multicolumn{2}{|c|}{$\begin{array}{c}0.0228 \\
(0.1893)\end{array}$} & \multicolumn{2}{|c|}{$\begin{array}{l}0.7829^{*} \\
(0.0945)\end{array}$} & \multicolumn{2}{|c|}{$\begin{array}{l}0.8994^{*} \\
(0.0665)\end{array}$} & \multicolumn{2}{|c|}{$\begin{array}{l}0.8495^{*} \\
(0.1644)\end{array}$} & \multicolumn{2}{|c|}{$\begin{array}{l}0.9499^{*} \\
(0.0198)\end{array}$} \\
\hline$d$ & & & & & $\begin{array}{l}9.147^{* * *} \\
(0.068)\end{array}$ & $\begin{array}{c}0.379 \\
(0.795)\end{array}$ & & & & & & & & & & \\
\hline$Q(10)$ & $\begin{array}{l}15.585 \\
{[0.112]}\end{array}$ & $\begin{array}{l}13.933 \\
{[0.176]}\end{array}$ & $\begin{array}{l}16.107 \\
{[0.097]}\end{array}$ & $\begin{array}{l}13.952 \\
{[0.175]}\end{array}$ & $\begin{array}{l}13.244 \\
{[0.210]}\end{array}$ & $\begin{array}{l}14.516 \\
{[0.151]}\end{array}$ & $\begin{array}{l}11.774 \\
{[0.300]}\end{array}$ & $\begin{array}{l}10.746 \\
{[0.378]}\end{array}$ & $\begin{array}{c}7.425 \\
{[0.685]}\end{array}$ & $\begin{array}{l}16.402 \\
{[0.089]}\end{array}$ & $\begin{array}{c}7.355 \\
{[0.692]}\end{array}$ & $\begin{array}{l}16.280 \\
{[0.092]}\end{array}$ & $\begin{array}{c}8.007 \\
{[0.628]}\end{array}$ & $\begin{array}{l}15.282 \\
{[0.122]}\end{array}$ & $\begin{array}{c}9.164 \\
{[0.517]}\end{array}$ & $\begin{array}{l}17.295 \\
{[0.068]}\end{array}$ \\
\hline$Q^{2}(10)$ & $\begin{array}{l}10.011 \\
{[0.264]}\end{array}$ & $\begin{array}{c}7.672 \\
{[0.466]}\end{array}$ & $\begin{array}{c}5.260 \\
{[0.729]}\end{array}$ & $\begin{array}{c}6.840 \\
{[0.554]}\end{array}$ & $\begin{array}{l}10.936 \\
{[0.205]}\end{array}$ & $\begin{array}{c}9.619 \\
{[0.293]}\end{array}$ & $\begin{array}{l}11.584 \\
{[0.171]}\end{array}$ & $\begin{array}{c}5.372 \\
{[0.717]}\end{array}$ & $\begin{array}{c}6.827 \\
{[0.555]}\end{array}$ & $\begin{array}{c}5.204 \\
{[0.736]}\end{array}$ & $\begin{array}{l}13.116 \\
{[0.108]}\end{array}$ & $\begin{array}{c}5.205 \\
{[0.735]}\end{array}$ & $\begin{array}{l}12.806 \\
{[0.119]}\end{array}$ & $\begin{array}{c}6.231 \\
{[0.621]}\end{array}$ & $\begin{array}{c}3.831 \\
{[0.872]}\end{array}$ & $\begin{array}{c}8.779 \\
{[0.361]}\end{array}$ \\
\hline $\mathrm{ARCH}(10)$ & $\begin{array}{c}1.153 \\
{[0.319]}\end{array}$ & $\begin{array}{c}0.790 \\
{[0.638]}\end{array}$ & $\begin{array}{c}0.732 \\
{[0.695]}\end{array}$ & $\begin{array}{c}0.720 \\
{[0.706]}\end{array}$ & $\begin{array}{c}1.015 \\
{[0.429]}\end{array}$ & $\begin{array}{c}0.999 \\
{[0.442]}\end{array}$ & $\begin{array}{c}1.240 \\
{[0.261]}\end{array}$ & $\begin{array}{c}0.606 \\
{[0.810]}\end{array}$ & $\begin{array}{c}0.692 \\
{[0.733]}\end{array}$ & $\begin{array}{c}0.562 \\
{[0.846]}\end{array}$ & $\begin{array}{c}1.424 \\
{[0.164]}\end{array}$ & $\begin{array}{c}0.559 \\
{[0.848]}\end{array}$ & $\begin{array}{c}1.417 \\
{[0.167]}\end{array}$ & $\begin{array}{c}0.659 \\
{[0.763]}\end{array}$ & $\begin{array}{c}0.458 \\
{[0.917]}\end{array}$ & $\begin{array}{c}0.895 \\
{[0.537]}\end{array}$ \\
\hline$J B$ & $\begin{array}{l}57.366 \\
{[0.000]}\end{array}$ & $\begin{array}{l}257.487 \\
{[0.000]}\end{array}$ & $\begin{array}{l}226.231 \\
{[0.000]}\end{array}$ & $\begin{array}{l}70.761 \\
{[0.000]} \\
\end{array}$ & $\begin{array}{l}548.157 \\
{[0.000]}\end{array}$ & $\begin{array}{l}152.922 \\
{[0.000]}\end{array}$ & $\begin{array}{l}49.460 \\
{[0.000]}\end{array}$ & $\begin{array}{l}68.624 \\
{[0.000]}\end{array}$ & $\begin{array}{l}68.290 \\
{[0.000]}\end{array}$ & $\begin{array}{l}290.504 \\
{[0.000]}\end{array}$ & $\begin{array}{l}154.267 \\
{[0.000]}\end{array}$ & $\begin{array}{l}61.534 \\
{[0.000]}\end{array}$ & $\begin{array}{l}1286.90 \\
{[0.000]}\end{array}$ & $\begin{array}{l}136.948 \\
{[0.000]}\end{array}$ & $\begin{array}{l}1010.29 \\
{[0.000]}\end{array}$ & $\begin{array}{l}42.135 \\
{[0.000]}\end{array}$ \\
\hline$\rho_{S O}^{m}$ & \multicolumn{2}{|c|}{0.0390} & \multicolumn{2}{|c|}{0.0050} & \multicolumn{2}{|c|}{-0.0113} & & & & & & 601 & & & & \\
\hline$A I C$ & & & 11. & & 11. & & & & & & & 538 & & 07 & & 70 \\
\hline
\end{tabular}

Note: *** and *** denote rejection of $\mathrm{HO}$ null hypothesis at 1\%, 5\% and 10\% significance levels, respectively. Q(10) and Q2(10) are the Ljung-Box tests for autocorrelations of order 10 for the returns and for the squared returns, respectively. ARCH(10) test is the statistical test for conditional heteroscedasticity of order 10. $\rho_{\text {so }}^{m}$ denotes average value of time-varying dynamic conditional correlation coefficient. 
Abioğlu, V. (2021), "Volatility Spillovers and Correlations between Oil Prices and Stock Sectors in Turkey: Implications on Portfolio Hedging and Diversification Opportunities", Sosyoekonomi, 29(47), 79-106.

\section{Figure: 1 \\ Dynamic Conditional Correlations}
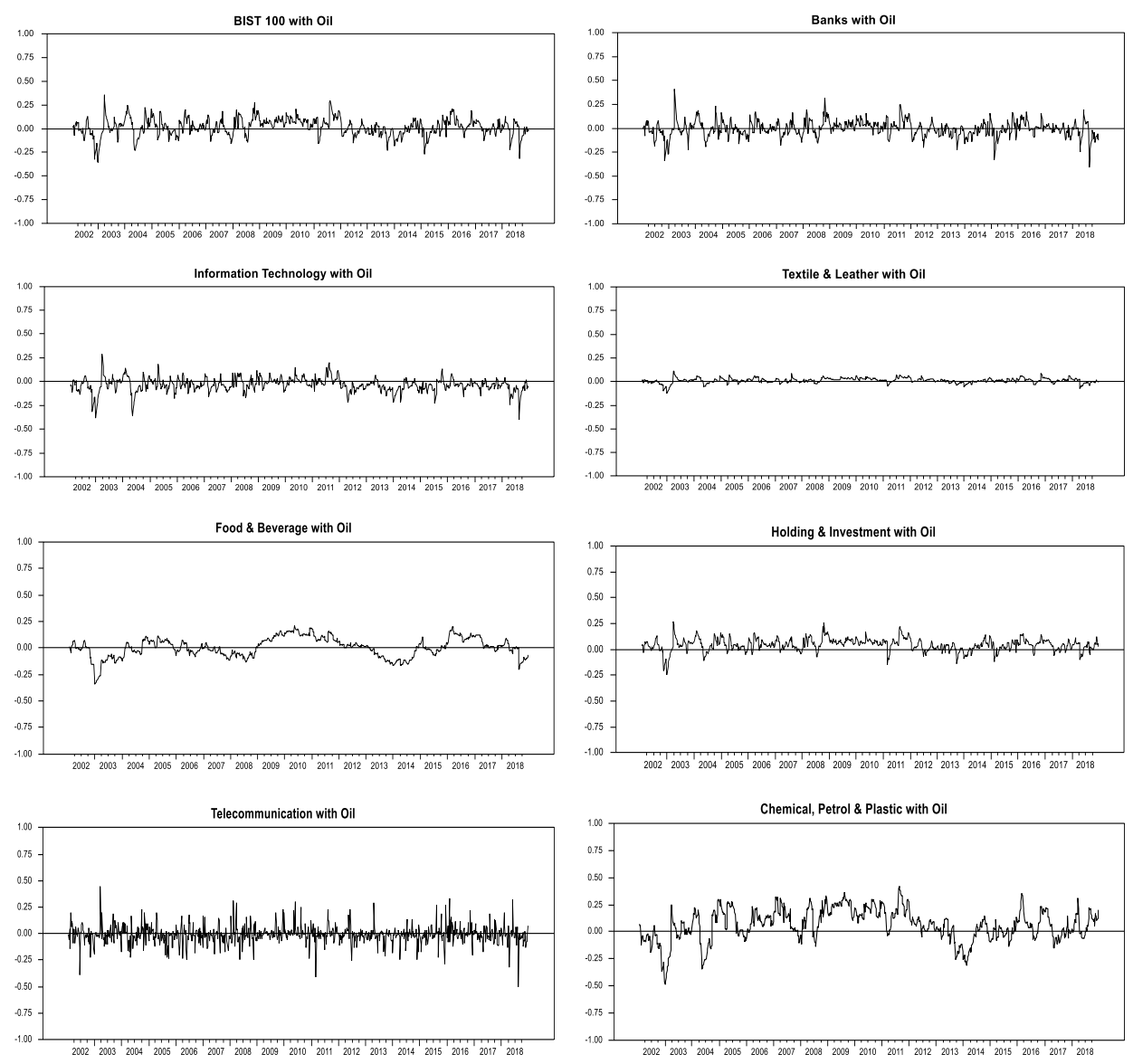
Abioğlu, V. (2021), "Volatility Spillovers and Correlations between Oil Prices and Stock Sectors in Turkey: Implications on Portfolio Hedging and Diversification Opportunities", Sosyoekonomi, 29(47), 79-106.
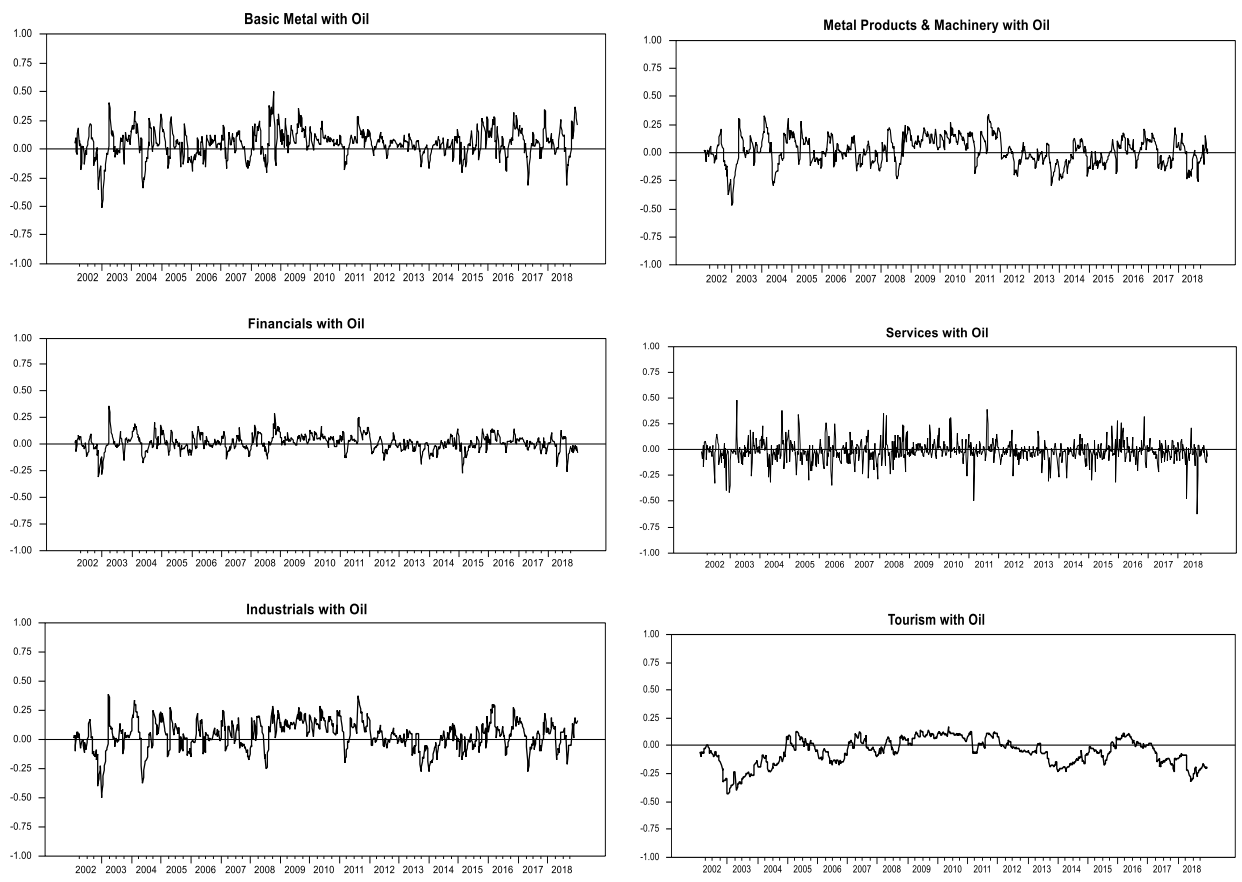

In summary, our findings reveal evidence of volatility spillovers from the oil market to the BIST 100 and twelve stock sectors. Moreover, degree of volatility spillover varies from one industry to another, mainly because some sectors are more oil-intensive than others. For example, Industrials and its sub-sectors Chemical, Petrol \& Plastic, Basic Metal, and Metal Products \& Machinery are relatively more oil-intensive sectors in their production process than others and are more closely related to oil price movements as seen from the dynamic correlation coefficients in Figure 1. However, the financial performance of these sectors can be affected differently from oil price hikes depending on whether these sectors are oil producing or oil consuming in their production. Further, volatilities of non-oilintensive sectors can also be affected by oil price changes since these sectors' main customer industries may depend on oil. Additionally, as oil price increases create an economy-wide recessionary effect by reducing total demand in the economy, oil price hikes can have a negative impact on non-oil-intensive sectors' financial performances to different degrees.

\subsection{Hedge Ratios, Optimal Portfolio Weights, Hedging and Diversification Effectiveness}

Kroner \& Sultan (1993) propose risk-minimizing hedge ratios in order to protect an asset against a certain risk. Company stocks can be considered for hedging against oil price fluctuations. If an oil asset and a company stock are positively correlated, a long position of one unit in a company's stock must be hedged by a short position of $\beta$ units in an oil asset 
to hedge against the risk of oil price fluctuations. On the other hand, if an oil asset and a company stock are negatively correlated, a long position of one unit in a company stock must be hedged by a long position of $\beta$ units in an oil asset to hedge against the risk of oil price fluctuations. In other words, a positive hedge ratio is the number of spot or future oil assets that an investor must sell for each unit of spot assets purchased, and a negative hedge ratio is the number of spot or future oil assets that an investor must buy for each unit of spot assets purchased. The optimal hedge ratio can then be estimated from an OLS regression of stock returns on oil returns (Ederington, 1979; Figlewski, 1985; Myers \& Thompson, 1989; Benet, 1992).

$$
r_{S, t}=\alpha+\beta r_{O, t}+\varepsilon_{t}
$$

where $r_{S, t}$ and $r_{O, t}$ denote returns on the stock index and oil price, respectively, and $\beta$ is the hedge ratio. The return on a portfolio of stock asset hedged using an oil asset can be represented as:

$$
r_{H, t}=r_{S, t}-\beta_{t} r_{O, t}
$$

where $r_{H, t}$ is the return of the hedged portfolio, $r_{S, t}$ is the stock return, $r_{O, t}$ is the oil return and $\beta_{t}$ is the hedge ratio; that is, the number of oil assets that an investor must sell for each unit of sector stock.

The variance of the return on the hedged portfolio conditional on the information set at time $(t-1)$ is given by:

$$
\operatorname{var}\left(r_{H, t} / \Omega_{t-1}\right)=\operatorname{var}\left(r_{S, t} / \Omega_{t-1}\right)-2 \beta_{t} \operatorname{cov}\left(r_{S, t}, r_{O, t} / \Omega_{t-1}\right)+\beta_{t}^{2} \operatorname{var}\left(r_{O, t} / \Omega_{t-1}\right)
$$

where $\operatorname{var}\left(r_{H, t} / \Omega_{t-1}\right)$ is the variance of hedged portfolio return, $\operatorname{var}\left(r_{s, t} / \Omega_{t-1}\right)$, $\operatorname{var}\left(r_{O, t} / \Omega_{t-1}\right)$ and $\operatorname{cov}\left(r_{S, t}, r_{O, t} / \Omega_{t-1}\right)$ are variances and covariance of stock and oil returns, respectively. From this equation, optimal hedge ratio can be obtained by taking the partial derivative of the portfolio variance with respect to $\beta_{t}$ and then setting this expression equal to zero and solving it for $\beta_{t}$, which yields:

$$
\beta_{t}=\frac{\operatorname{cov}\left(r_{S, t}, r_{O, t} / \Omega_{t-1}\right)}{\operatorname{var}\left(r_{O, t} / \Omega_{t-1}\right)}
$$

Thus, the optimal hedge ratio, $\beta_{t}$, which is the number of oil assets an investor must sell for each unit of sector stock, minimizes the variance of the hedged portfolio returns.

Considering the time-varying variance-covariance structure of financial variables, many researchers use multivariate conditional volatility models to calculate hedge ratios (Baillie \& Myers, 1991; Kroner \& Sultan, 1993; Ku et al., 2007). Given the multivariate 
conditional volatility model, an estimated conditional variance-covariance matrix can be used to calculate the hedge ratio $\beta_{t}$ as follows (Kroner \& Sultan, 1993):

$$
\boldsymbol{\beta}_{\text {SO,t }}=\frac{\boldsymbol{h}_{\text {SO,t }}}{\boldsymbol{h}_{O, t}}
$$

where $h_{S O, t}$ denotes the conditional covariance between stock and oil returns and $h_{O, t}$ is the conditional variance of the oil return. Following Ku et al. (2007), the hedging effectiveness (HE) index can be calculated as follows:

$$
H E=\frac{\operatorname{var}_{u h}-\operatorname{var}_{h}}{\operatorname{var}_{u h}}
$$

where $\operatorname{var}_{u h}$ is the variance of the returns $\left(r_{S, t}\right)$ on the unhedged portfolio which consists of only sector stocks, and $\mathrm{var}_{h}$ is the variance of the hedged portfolio returns $\left(r_{H, t}\right)$. Higher HE index values indicate better hedging effectiveness and larger risk reduction for hedged portfolios. On the other hand, negative hedging effectiveness values indicate hedged portfolios are worse than unhedged portfolios. Hedging effectiveness can be used to compare the performance different multivariate conditional volatility models. A better conditional volatility model should be superior in terms of hedging effectiveness as it largely reduces the variance of a hedged portfolio.

Alternatively, an optimal portfolio design can be constructed using optimal portfolio weights that minimize risk without lowering expected returns (Kroner \& Ng, 1998; Hammoudeh et al., 2010). Suppose $w_{S o, t}$ is a weight of oil asset in a one-dollar portfolio. As such, $\left(1-w_{s o, t}\right)$ is the weight of stock market (sector) in the one-dollar oil-stock portfolio.

$$
r_{W, t}=w_{S O, t} \times r_{O, t}+\left(1-w_{S O, t}\right) \times r_{S, t}
$$

where $r_{W, t}$ denotes the return on the weighted oil-stock portfolio. Based on equation (30), the variance of the return on the oil-stock portfolio conditional on the information set at time $(t-1)$ is given by:

$$
\begin{aligned}
\operatorname{var}\left(r_{W, t} / \Omega_{t-1}\right)= & w_{S O, t}^{2} \operatorname{var}\left(r_{O, t} / \Omega_{t-1}\right)+2 w_{S O, t}\left(1-w_{S O, t}\right) \operatorname{cov}\left(r_{O, t}, r_{S, t} / \Omega_{t-1}\right)+ \\
& +\left(1-w_{S O, t}\right)^{2} \operatorname{var}\left(r_{S, t} / \Omega_{t-1}\right)
\end{aligned}
$$

where $\operatorname{var}\left(r_{W, t} / \Omega_{t-1}\right)$ is the variance of the return on the weighted oil-stock portfolio, $\operatorname{var}\left(r_{S, t} / \Omega_{t-1}\right), \operatorname{var}\left(r_{O, t} / \Omega_{t-1}\right)$ and $\operatorname{cov}\left(r_{S, t}, r_{O, t} / \Omega_{t-1}\right)$ are variances and covariance of stock and oil returns respectively. Thus, the optimal weight that minimizes variance of the oil-stock portfolio $\left(\operatorname{var}\left(r_{W, t} / \Omega_{t-1}\right)\right)$ can be obtained by taking the partial derivative 
of the oil-stock portfolio variance with respect to $w_{S O, t}$ and then setting this expression equal to zero and solving for $w_{S O, t}$, which yields:

$$
w_{S O, t}=\frac{\operatorname{var}\left(r_{S, t} / \Omega_{t-1}\right)-\operatorname{cov}\left(r_{O, t}, r_{S, t} / \Omega_{t-1}\right)}{\operatorname{var}\left(r_{S, t} / \Omega_{t-1}-2 \operatorname{cov}\left(r_{O, t}, r_{S, t} / \Omega_{t-1}\right)+\operatorname{var}\left(r_{O, t} / \Omega_{t-1}\right.\right.}
$$

where $w_{S O, t}$ minimizes conditional variance of the oil-stock portfolio. Given the multivariate conditional volatility model, an estimated conditional variance-covariance matrix can be used to calculate risk-minimizing optimal oil weight (Kroner \& $\mathrm{Ng}, 1998$ ).

$$
\begin{aligned}
& w_{S O, t}=\frac{h_{S, t}-h_{S O, t}}{h_{S, t}-2 h_{S O, t}+h_{O, t}} \\
& w_{S O, t}=\left\{\begin{array}{ccc}
0, & \text { if } & w_{S O, t}<0 \\
w_{S O, t}, & \text { if } & 0 \leq w_{S O, t} \leq 1 \\
1, & \text { if } & w_{S O, t}>1
\end{array}\right.
\end{aligned}
$$

Using the four alternative MGARCH models presented in Section 2, hedge ratios, hedging effectiveness, optimal portfolio weights and effectiveness of diversification for all sectors are computed and compared to determine which MGARCH model works best for hedging stock sectors. Table 3 reports the average values of time-varying hedge ratios and optimal weights for each model. The average hedge ratios range from -0.11 to 0.06 in the CCC and CCC-AGARCH models, while they range from -0.08 to 0.06 in the DCC and ADCC models. The results from all four models suggest that Chemical, Petrol \& Plastic, Basic Metal and Insurance have high average hedge ratios, while Transportation, Tourism and Information Technology have high negative-average hedge ratios. Since the hedge ratios are time-varying, investors in stock markets should change their long and short positions according to the time-varying hedge ratios. Positive hedge ratios imply positive correlations between oil and stock assets, and for these pairs of assets long positions in stock assets should be shorted by oil assets. On the other hand, negative hedge ratios indicate that there are negative correlations between oil and stock assets, and for these pairs of assets long positions should be taken in each asset. For example, an 0.0570 average hedge ratio between Chemical, Petrol \& Plastic stocks and oil assets from the DCC model suggest that a 1 TL long position in a Chemical, Petrol \& Plastic stock should be hedged by a 0.0570 TL short position in an oil asset. Similarly, a -0.0841 hedge ratio between a Transportation stock and an oil asset suggests that a 1 TL long position in Transportation should be hedged by a 0.0841 TL long position in an oil asset. Furthermore, the findings show that average hedge ratios are generally low for most sectors. In particular, the CCC and CCC-AGARCH models suggest that average hedge ratios for the BIST 100, Textile \& Leather, Holding \& Investments, Wood, Paper \& Printing, Metal Product \& Machinery, Technology, Securities Investment Trusts and REITs are nearly zero, while the DCC and ADCC models suggest that hedge ratios for Banks, Food \& Beverage, Textile \& Leather, Technology and REITs 
Abioğlu, V. (2021), "Volatility Spillovers and Correlations between Oil Prices and Stock Sectors in Turkey: Implications on Portfolio Hedging and Diversification Opportunities", Sosyoekonomi, 29(47), 79-106.

are nearly zero. These values imply that, on average, fewer oil assets are needed to minimize the risk of these stock holdings.

Table: 3

Average Hedge Ratios and Average Portfolio Weights for Oil-Stock Portfolios

\begin{tabular}{|c|c|c|c|c|c|c|c|c|}
\hline & \multicolumn{2}{|c|}{ CCC } & \multicolumn{2}{|c|}{ CCC-AGARCH } & \multicolumn{2}{|c|}{ DCC } & \multicolumn{2}{|c|}{ ADCC } \\
\hline & $H R$ & $w_{S O}$ & $H R$ & $w_{S O}$ & $H R$ & $w_{S O}$ & $H R$ & $w_{S O}$ \\
\hline BIST 100 & -0.0051 & 0.4832 & -0.0055 & 0.4874 & 0.0139 & 0.4826 & 0.0136 & 0.4826 \\
\hline Banks & -0.0269 & 0.6125 & -0.0300 & 0.6129 & -0.0081 & 0.6145 & -0.0034 & 0.6151 \\
\hline Information Technology & -0.0655 & 0.5462 & -0.0664 & 0.5485 & -0.0407 & 0.5502 & -0.0407 & 0.5502 \\
\hline Textile \& Leather & 0.0023 & 0.4670 & 0.0018 & 0.4719 & 0.0098 & 0.4663 & 0.0095 & 0.4669 \\
\hline Food \& Beverage & -0.0128 & 0.4739 & -0.0142 & 0.4783 & 0.0029 & 0.4733 & -0.0014 & 0.4721 \\
\hline Holding \& Investment & 0.0024 & 0.5171 & 0.0011 & 0.5200 & 0.0432 & 0.5182 & 0.0272 & 0.5203 \\
\hline Telecommunication & -0.0213 & 0.5434 & -0.0201 & 0.5490 & -0.0107 & 0.5436 & -0.0107 & 0.5436 \\
\hline Wood, Paper \& Printing & -0.0074 & 0.5101 & -0.0051 & 0.5086 & 0.0113 & 0.5102 & 0.0108 & 0.5096 \\
\hline Chemical, Petrol \& Plastic & 0.0582 & 0.4796 & 0.0577 & 0.4833 & 0.0570 & 0.4762 & 0.0654 & 0.4760 \\
\hline Basic Metal & 0.0341 & 0.5865 & 0.0225 & 0.5884 & 0.0585 & 0.5900 & 0.0587 & 0.5924 \\
\hline Metal Products \& Machinery & -0.0058 & 0.4929 & -0.0047 & 0.4953 & 0.0148 & 0.4931 & 0.0142 & 0.4932 \\
\hline Insurance & 0.0320 & 0.5008 & 0.0412 & 0.4927 & 0.0399 & 0.5001 & 0.0497 & 0.5006 \\
\hline Securities Investment Trusts & 0.0097 & 0.4633 & 0.0121 & 0.4854 & 0.0273 & 0.4628 & 0.0388 & 0.4622 \\
\hline Electricity & -0.0133 & 0.5541 & -0.0141 & 0.5557 & -0.0016 & 0.5541 & 0.0113 & 0.5538 \\
\hline Financials & -0.0166 & 0.5605 & -0.0175 & 0.5636 & 0.0116 & 0.5625 & 0.0136 & 0.5627 \\
\hline Services & -0.0250 & 0.4059 & -0.0266 & 0.4101 & -0.0158 & 0.4041 & -0.0110 & 0.4036 \\
\hline Industrials & 0.0198 & 0.4096 & 0.0190 & 0.4139 & 0.0291 & 0.4064 & 0.0308 & 0.4059 \\
\hline Technology & -0.0075 & 0.5423 & -0.0128 & 0.5508 & 0.0049 & 0.5429 & 0.0083 & 0.5432 \\
\hline Leasing \& Factoring & -0.0280 & 0.5956 & -0.0239 & 0.6096 & -0.0134 & 0.5977 & -0.0228 & 0.5974 \\
\hline Wholesale \& Retail Trade & -0.0244 & 0.4396 & -0.0233 & 0.4402 & -0.0128 & 0.4386 & -0.0087 & 0.4520 \\
\hline Non-Metal Mineral Products & -0.0329 & 0.4005 & -0.0317 & 0.4029 & -0.0201 & 0.3987 & -0.0176 & 0.3986 \\
\hline Transportation & -0.1141 & 0.6122 & -0.1134 & 0.6153 & -0.0841 & 0.6144 & -0.0818 & 0.6147 \\
\hline Real Estate Investment Trusts & -0.0094 & 0.5071 & 0.0054 & 0.5185 & 0.0082 & 0.5077 & -0.0003 & 0.5075 \\
\hline Tourism & -0.0955 & 0.6163 & -0.0971 & 0.6163 & -0.0831 & 0.6180 & -0.0838 & 0.6179 \\
\hline
\end{tabular}

Note: $\beta_{\text {so }}$ denotes average hedge ratio and $w_{\text {so denotes average weight of oil assets in a one-dollar oil-stock }}$ portfolio.

Table 3 also reports the optimal weights of oil in oil-stock portfolios that minimize the risk (variance) of the portfolios without lowering the expected returns. Optimal portfolio weights calculated from all four models are not particularly different from each other, suggesting that portfolio compositions give similar results for each MGARCH model. By sector, the optimal weight of oil in oil-stock portfolios ranges from nearly $40 \%$ (Non-Metal Mineral Products, Services and Industrials) to nearly 62\% (Tourism, Transportation and Banks) for each model. These imply that for the Non-Metal Mineral Products sector the optimal allocation of oil in a $1 \mathrm{TL}$ oil-stock portfolio should be $0.40 \mathrm{TL}$ with the remaining 0.60 TL invested in Non-Metal Mineral Products; while for the Tourism sector, the optimal allocation of oil should be $0.62 \mathrm{TL}$ with the remaining $0.38 \mathrm{TL}$ invested in Tourism. According to all four models, the optimal weight of oil in oil-stock portfolios is less than $50 \%$ for ten sectors (BIST100; Food and Beverage; Textile \& Leather; Chemical, Petrol \& Plastic; Metal Products \& Machinery; Securities Investment Trusts; Services; Industrials; Wholesale \& Retail Trade and Non-Metal Mineral Products), while for the other thirteen sectors optimal weights of oil are higher than $50 \%$. In summary, our findings on portfolio diversification show that in order to minimize risk of an oil-stock portfolio without lowering the expected return, investors should hold more stocks than oil assets in their oil-stock portfolio for ten sectors, while for the remaining fourteen sectors, investors should hold less stocks than oil assets in their oil-stock portfolios. 
Abioğlu, V. (2021), "Volatility Spillovers and Correlations between Oil Prices and Stock Sectors in Turkey: Implications on Portfolio Hedging and Diversification Opportunities", Sosyoekonomi, 29(47), 79-106.

Table: 4

Hedging and Diversification Effectiveness (\%)

\begin{tabular}{|c|c|c|c|c|c|c|c|c|}
\hline & \multicolumn{2}{|c|}{$\mathrm{CCC}$} & \multicolumn{2}{|c|}{ CCC-AGARCH } & \multicolumn{2}{|c|}{ DCC } & \multicolumn{2}{|c|}{ ADCC } \\
\hline & $H E$ & $D E$ & $H E$ & $D E$ & $H E$ & $D E$ & $H E$ & $D E$ \\
\hline BIST 100 & 2.72 & 48.10 & 2.71 & 48.55 & 4.38 & 47.98 & 4.40 & 47.97 \\
\hline Banks & 2.47 & 61.71 & 2.52 & 62.19 & 3.60 & 61.78 & 3.52 & 61.77 \\
\hline Information Technology & 2.52 & 56.81 & 2.51 & 57.36 & 3.53 & 56.76 & 3.53 & 56.76 \\
\hline Textile \& Leather & 3.42 & 46.53 & 3.42 & 47.26 & 3.77 & 46.51 & 3.70 & 46.71 \\
\hline Food \& Beverage & 1.84 & 45.54 & 1.85 & 45.38 & 2.69 & 45.61 & 2.83 & 45.63 \\
\hline Holding \& Investment & 2.25 & 51.40 & 2.23 & 51.80 & 3.92 & 51.37 & 3.94 & 51.16 \\
\hline Telecommunication & 6.17 & 59.78 & 6.17 & 60.46 & 6.20 & 59.82 & 6.20 & 59.82 \\
\hline Wood, Paper \& Printing & 1.39 & 49.43 & 1.39 & 49.99 & 1.84 & 49.38 & 2.12 & 49.41 \\
\hline Chemical, Petrol \& Plastic & 2.78 & 45.14 & 2.83 & 45.64 & 5.12 & 45.12 & 4.90 & 45.20 \\
\hline Basic Metal & 1.94 & 55.44 & 1.76 & 55.45 & 3.96 & 55.66 & 3.99 & 55.56 \\
\hline Metal Products \& Machinery & 1.16 & 48.98 & 1.16 & 49.27 & 3.83 & 48.90 & 3.85 & 48.89 \\
\hline Insurance & 0.73 & 56.21 & 0.66 & 56.91 & 2.00 & 56.16 & 2.01 & 56.10 \\
\hline Securities Investment Trusts & 1.68 & 50.40 & 1.52 & 48.13 & 2.86 & 50.43 & 2.35 & 50.51 \\
\hline Electricity & 1.32 & 55.90 & 1.33 & 56.16 & 1.72 & 56.01 & 1.53 & 55.94 \\
\hline Financials & 2.42 & 56.40 & 2.42 & 56.81 & 3.81 & 56.39 & 3.75 & 56.39 \\
\hline Services & 5.89 & 43.65 & 5.85 & 44.45 & 6.76 & 43.19 & 6.70 & 43.35 \\
\hline Industrials & 2.05 & 38.48 & 2.08 & 38.52 & 4.50 & 38.28 & 4.37 & 38.33 \\
\hline Technology & 1.86 & 53.60 & 1.76 & 53.22 & 2.39 & 53.59 & 2.59 & 53.62 \\
\hline Leasing \& Factoring & 0.80 & 67.45 & 0.74 & 69.30 & 0.80 & 67.41 & 1.14 & 67.41 \\
\hline Wholesale \& Retail Trade & 2.17 & 45.39 & 2.13 & 46.23 & 2.49 & 45.29 & 2.32 & 46.38 \\
\hline Non-Metal Mineral Products & 0.64 & 40.52 & 0.67 & 40.98 & 1.99 & 40.43 & 1.97 & 40.46 \\
\hline Transportation & 1.25 & 63.01 & 1.29 & 63.53 & 1.89 & 63.05 & 1.92 & 63.03 \\
\hline Real Estate Investment Trusts & 1.59 & 52.53 & 1.70 & 53.00 & 2.47 & 52.44 & 2.63 & 52.40 \\
\hline Tourism & 5.61 & 66.49 & 5.71 & 66.81 & 8.92 & 66.59 & 8.91 & 66.60 \\
\hline
\end{tabular}

Note: $H E$ and DE denote hedging effectiveness and diversification effectiveness, respectively.

Table 4 reports hedging effectiveness (HE) of hedged portfolios constructed using hedge ratios. Hedging effectiveness ratios are calculated by comparing variances of a hedged portfolio and an unhedged portfolio as shown in equation (18). A positive HE ratio implies that the hedged portfolio is better than the unhedged portfolio in terms of the hedged portfolio's variance reduction, while a negative HE ratio implies that the hedged portfolio is worse than the unhedged portfolio. As seen from Table 4, for DCC and ADCC models, variance reductions range from $0.80 \%$ (Leasing \& Factoring) to $8.92 \%$ (Tourism), while for CCC and CCC-AGARCH models the variance reductions range from $0.64 \%$ (Non-Metal Mineral Products) to $6.17 \%$ (Telecommunication). Hence, DCC and ADCC models are more effective than CCC and CCC-AGARCH models in reducing variances of hedged portfolios for all sectors. Since time-varying hedge ratios are closely related to time-varying correlations, DCC and ADCC models provide more accurate estimates of time-varying hedge ratios. However, CCC and CCC-AGARCH models cannot reduce risks (variances) of hedged portfolios as effectively as DCC and ADCC models, as they assume constant correlation coefficient between asset returns (e.g., Sadorsky, 2012; Lin et al., 2014; Ahmad et al., 2018). Further, according to the hedging effectiveness results of the DCC and ADCC models, the portfolio variance is reduced most significantly for the following sectors, with results shown for each model, respectively: Tourism (8.92\% and $8.91 \%)$, Services $(6.76 \%$ and $6.70 \%)$, Telecommunication (6.20\% and 6.20\%), Chemical, Petrol \& Plastic (5.12\% and $4.90 \%)$, Industrials (4.50\% and $4.37 \%)$ and the BIST 100 (4.38\% and $4.40 \%)$.

Diversification effectiveness (DE) of weighted oil-stock portfolios constructed using optimal weight for oil is reported in Table 4. Like the HE values of hedged portfolios, the effectiveness of portfolio diversification is calculated by comparing variances of a weighted 
oil-stock portfolio and an unhedged portfolio. A higher DE value implies a greater effectiveness of portfolio diversification in terms of a diversified portfolio's variance reduction. The results indicate high diversification effectiveness ratios for all sectors in each of the MGARCH models. Furthermore, variance reductions remain highly stable across MGARCH models and range from nearly 38\% (Industrials) to 66\% (Tourism). Also, DE values are higher than $50 \%$ for most of the sectors. These results indicate that diversification is a more effective strategy than hedging in reducing variance of an oil-stock portfolio. It is obvious that negative correlations (i.e., negative hedge ratios) between crude oil and sector returns provide better diversification opportunities for stock sectors. Further, close-to-zero correlations between oil and stock sectors also provide better diversification opportunities for investors as the risk of an asset can be used to offset the risk of another asset without reducing the expected return of an oil-stock portfolio. Due to these reasons, it can be said that diversification is more effective than a hedging strategy at reducing variance of oil-stock portfolios in Turkish stock markets.

Next, following Arouri et al. (2011), portfolio return simulations are run by calculating risk-adjusted returns of hedged and diversified portfolios and comparing them with the risk-adjusted return of unhedged portfolios for all sectors. Risk-adjusted return of a portfolio ( $\mathrm{rar}$ ) is measured by calculating the ratio of the portfolio's mean return to its standard deviation. Unhedged portfolios are those composed of $100 \%$ stocks. The results are shown in Table 5. Figures in boldface indicate that the risk-adjusted returns of hedged and diversified portfolios are higher than the risk-adjusted returns of unhedged portfolios. The results indicate that, in $\mathrm{CCC}$ and $\mathrm{CCC}$-AGARCH models, risk-adjusted returns of hedged portfolios are higher than risk-adjusted returns of unhedged portfolios for all sectors. Further, in DCC and ADCC models, risk-adjusted return of hedged portfolios is higher than risk-adjusted return of unhedged portfolios for all sectors except for Food \& Beverage. Lower risk-adjusted return of Food \& Beverage may stem from the diminishing unconditional mean return value of hedged portfolios. Table 5 also reveals that the riskadjusted return of weighted oil-stock portfolios is higher than the risk-adjusted return of unhedged portfolios for all sectors in each of the MGARCH models. Hence, the results from portfolio simulations in Table 5 show that both strategies improve risk-adjusted returns of oil-stock portfolios for all sectors. Also, the risk-adjusted return of diversified portfolios is relatively higher than the risk-adjusted return of hedged portfolios for all sectors except for Basic Metal in DCC and ADCC models. Thus, risk-adjusted performances of diversified portfolios indicate that portfolio diversification is a more effective strategy than portfolio hedging for Turkish stock markets. 
Table: 5

Risk-Adjusted Returns of Hedging and Diversification (\%)

\begin{tabular}{|c|c|c|c|c|c|c|c|c|c|c|c|c|}
\hline & \multicolumn{3}{|c|}{ CCC } & \multicolumn{3}{|c|}{ CCC-AGARCH } & \multicolumn{3}{|c|}{ DCC } & \multicolumn{3}{|c|}{ ADCC } \\
\hline & $\operatorname{rar}_{H}$ & $\operatorname{rar}_{D}$ & $r a r_{U H}$ & $\operatorname{rar}_{H}$ & $\operatorname{rar}_{D}$ & $\operatorname{rar}_{U H}$ & $\operatorname{rar}_{H}$ & $r a r_{D}$ & $\operatorname{rar}_{U H}$ & $\operatorname{rar}_{H}$ & $\operatorname{rar}_{D}$ & $\operatorname{rar}_{U H}$ \\
\hline BIST 100 & 6.19 & 7.73 & 5.56 & 6.20 & 7.84 & 5.56 & 6.78 & 7.64 & 5.56 & 6.74 & 7.64 & 5.56 \\
\hline Banks & 4.21 & 6.44 & 3.75 & 4.23 & 6.28 & 3.75 & 4.67 & 6.40 & 3.75 & 4.91 & 6.37 & 3.75 \\
\hline Information Technology & 2.48 & 5.42 & 1.49 & 2.50 & 6.03 & 1.49 & 2.64 & 5.37 & 1.49 & 2.64 & 5.37 & 1.49 \\
\hline Textile \& Leather & 5.61 & 8.13 & 5.04 & 5.61 & 8.77 & 5.04 & 5.76 & 8.10 & 5.04 & 5.49 & 8.12 & 5.04 \\
\hline Food \& Beverage & 6.32 & 8.25 & 5.87 & 6.34 & 8.75 & 5.87 & 5.51 & 8.15 & 5.87 & 5.24 & 8.18 & 5.87 \\
\hline Holding \& Investment & 4.80 & 6.64 & 4.40 & 4.81 & 6.93 & 4.40 & 5.16 & 6.54 & 4.40 & 4.87 & 6.54 & 4.40 \\
\hline Telecommunication & 4.69 & 6.94 & 3.84 & 4.70 & 7.87 & 3.84 & 5.28 & 6.87 & 3.84 & 5.28 & 6.87 & 3.84 \\
\hline Wood, Paper \& Printing & 4.38 & 6.35 & 4.25 & 4.37 & 8.25 & 4.25 & 4.34 & 6.34 & 4.25 & 4.96 & 6.29 & 4.25 \\
\hline Chemical, Petrol \& Plastic & 7.63 & 9.41 & 6.60 & 7.61 & 9.54 & 6.60 & 7.69 & 9.31 & 6.60 & 7.82 & 9.35 & 6.60 \\
\hline Basic Metal & 8.43 & 9.20 & 7.91 & 8.46 & 9.64 & 7.91 & 9.80 & 9.16 & 7.91 & 9.83 & 9.17 & 7.91 \\
\hline Metal Products \& Machinery & 6.31 & 7.32 & 5.95 & 6.31 & 7.83 & 5.95 & 6.81 & 7.23 & 5.95 & 6.80 & 7.23 & 5.95 \\
\hline Insurance & 7.01 & 8.60 & 6.65 & 6.93 & 9.78 & 6.65 & 7.50 & 8.48 & 6.65 & 7.90 & 8.54 & 6.65 \\
\hline Securities Investment Trusts & 3.33 & 6.08 & 2.98 & 3.29 & 6.40 & 2.98 & 3.46 & 5.95 & 2.98 & 3.93 & 5.96 & 2.98 \\
\hline Electricity & 0.95 & 3.67 & 0.63 & 0.97 & 4.44 & 0.63 & 1.09 & 3.62 & 0.63 & 1.47 & 3.61 & 0.63 \\
\hline Financials & 4.69 & 6.60 & 4.21 & 4.70 & 6.58 & 4.21 & 5.12 & 6.53 & 4.21 & 5.25 & 6.52 & 4.21 \\
\hline Services & 8.45 & 9.77 & 7.27 & 8.48 & 10.37 & 7.27 & 9.15 & 9.78 & 7.27 & 9.74 & 9.81 & 7.27 \\
\hline Industrials & 8.25 & 9.35 & 7.57 & 8.25 & 9.37 & 7.57 & 8.83 & 9.26 & 7.57 & 8.86 & 9.27 & 7.57 \\
\hline Technology & 7.07 & 9.06 & 6.25 & 7.08 & 9.59 & 6.25 & 7.07 & 9.00 & 6.25 & 7.55 & 8.97 & 6.25 \\
\hline Leasing \& Factoring & 7.09 & 8.80 & 6.58 & 7.11 & 11.44 & 6.58 & 6.78 & 8.80 & 6.58 & 6.86 & 8.78 & 6.58 \\
\hline Wholesale \& Retail Trade & 9.57 & 10.62 & 8.52 & 9.57 & 10.66 & 8.52 & 9.67 & 10.60 & 8.52 & 9.54 & 10.11 & 8.52 \\
\hline Non-Metal Mineral Products & 6.29 & 7.68 & 5.64 & 6.31 & 8.29 & 5.64 & 6.41 & 7.72 & 5.64 & 6.51 & 7.74 & 5.64 \\
\hline Transportation & 7.01 & 7.77 & 6.14 & 7.05 & 8.41 & 6.14 & 6.60 & 7.64 & 6.14 & 6.64 & 7.62 & 6.14 \\
\hline Real Estate Investment Trusts & 2.61 & 5.32 & 1.82 & 2.53 & 6.87 & 1.82 & 2.90 & 5.28 & 1.82 & 2.51 & 5.38 & 1.82 \\
\hline Tourism & 2.11 & 5.84 & 1.84 & 2.16 & 6.27 & 1.84 & 2.03 & 5.76 & 1.84 & 1.99 & 5.77 & 1.84 \\
\hline
\end{tabular}

Note: rar $_{H}$ denotes risk-adjusted return of a hedged portfolio, rar $_{D}$ denotes risk-adjusted return of a diversified portfolio and rar ${ }_{U H}$ denotes risk-adjusted return of an unhedged portfolio. Figures in boldface indicate higher risk-adjusted returns of hedged and diversified portfolios than the risk-adjusted returns of unhedged portfolios 
Abioğlu, V. (2021), "Volatility Spillovers and Correlations between Oil Prices and Stock Sectors in Turkey: Implications on Portfolio Hedging and Diversification Opportunities", Sosyoekonomi, 29(47), 79-106.

\section{Conclusion}

This study investigates volatility spillover effects as well as hedging and diversification opportunities between sectoral stock returns and world crude oil prices. For this purpose, weekly closing prices of the BIST 100 and twenty-three sectoral stock indices are used and the DCC model is employed to investigate volatility spillovers between sectoral stock returns and world crude oil prices. Findings from the DCC model reveal significant volatility spillovers from the oil market to the BIST 100 and twelve stock sectors: Banks; Information Technology; Textile \& Leather; Food \& Beverage; Holding \& Investments; Telecommunication; Chemical, Petrol \& Plastic; Basic Metal; Metal Products \& Machinery; Financials; Services; and Industrials. That is, volatility spillovers from the oil market are found to increase volatilities in these sectors. Additionally, the degree of volatility transmission varies from one industry to another. This is primarily because some sectors are more oil-intensive than others. For example, Industrials and its sub-sectors of Chemical, Petrol \& Plastic, Basic Metal, and Metal Products \& Machinery are relatively more oilintensive in their production processes than others and are more closely correlated with oil price movements as can be seen from significant dynamic correlation coefficients. Further, financial performance of these sectors can also be affected differently from oil price hikes depending on whether these sectors are oil producing or oil consuming in their production. On the other hand, volatilities of non-oil-intensive sectors (such as Banks, Information Technology, Telecommunication, Financials and Services) can be affected differently by oil price changes since these sectors' main customer industries may depend on oil. For example, Lee \& Ni (2002) find that for industries that are oil intensive in production, such as petroleum refining and industrial chemical production, the main effects of oil shocks are on the supply side, while for other industries, the automobile industry in particular, the chief effects of oil price shocks are on the demand side. Also, Gogineni (2010) finds that the sensitivity of industries' returns to changes in oil price depends on both the cost-side and demand-side dependence on oil, and that the relative effects of these factors vary across industries. Additionally, as oil price increases create economy-wide recessionary effects by reducing total demand in the economy, oil price hikes can negatively impact the financial performance of non-oil-intensive sectors to different degrees. DCC estimation results also indicate that dynamic conditional correlations between oil and sectoral stock returns are generally at low levels and close-to-zero for some sectors.

Furthermore, four multivariate GARCH models, namely, CCC, CCC-AGARCH, DCC and ADCC, are employed to compute and compare optimal hedge ratios, optimal portfolio weights, hedging effectiveness, diversification effectiveness and risk-adjusted returns of oil-stock portfolios. The results indicate that DCC and ADCC models are the best in terms of the variance reduction in hedged portfolios for all sectors. Since time-varying hedge ratios are closely related to time-varying correlations, DCC and ADCC models provide more accurate estimates of time-varying hedge ratios. Hence, DCC and ADCC models can effectively reduce the variances of hedged portfolios for all sectors. Additionally, diversification is found to be a more effective strategy than hedging in terms of variance reductions and risk-adjusted returns in oil-stock portfolios. This is because negative correlations or negative hedge ratios between crude oil and sector returns provide 
Abioğlu, V. (2021), "Volatility Spillovers and Correlations between Oil Prices and Stock Sectors in Turkey: Implications on Portfolio Hedging and Diversification Opportunities", Sosyoekonomi, 29(47), 79-106.

better diversification opportunities between the oil and stock sectors. Further, close-to-zero correlations between the oil and stock sectors also provide better diversification opportunities for investors as the risk of an asset can be used to offset the risk of another asset without reducing the expected return of the oil-stock portfolio. For these reasons, it can be concluded that, although hedged portfolios are better than unhedged portfolios in terms of variance reductions and risk-adjusted returns, diversification works better than the hedging strategy in reducing variances of oil-stock portfolios and increasing risk-adjusted returns in Turkish stock markets. This implies that for the Turkish stock market, the diversification strategy is more profitable and less risky than the hedging strategy for investors having sufficient capital for diversification. However, if investors have insufficient capital to diversify their portfolios, a hedging strategy is still more profitable and less risky than unhedged portfolios for all sectors.

While the findings in this study are important for investors, portfolio managers and policy makers, the analyses can be extended in future research by using more sophisticated spectral or wavelet decomposition analysis to investigate risks and spillovers at different frequencies of data. Also, this study can be extended in the future to analyze potential financial and macroeconomic drivers of hedged portfolio returns that could affect the hedging dynamics in the Turkish stock market. In particular, since this study finds that there are potential benefits obtained from hedging and diversification strategies, it is necessary to identify potential financial and macroeconomic factors that drive risks and uncertainties in hedging and diversification.

\section{References}

Abdioğlu, Z. \& N. Değirmenci (2014), "The Relationship between Oil Prices and Stock Prices: BIST Sectoral Analysis", Kafkas University Journal of Economics and Administrative Sciences Faculty, 5(8), 1-24.

Ahmad, W. \& P. Sadorsky \& A. Sharma (2018), "Optimal hedge ratios for clean energy equities", Economic Modelling, 72, 278-295.

Aielli, G.P. (2013), "Dynamic Conditional Correlation: On Properties and Estimation", Journal of Business \& Economic Statistics, 31(3), 282-299.

Aktaş, M. \& S. Akdağ (2013), “Türkiye'de Ekonomik Faktörlerin Hisse Senedi Fiyatları ile İlişkilerinin Araştırılması”, International Journal of Social Science Research, 2(1), 5067.

Al-Fayoumi, N.A. (2009), "Oil Prices and Stock Market Returns in Oil Importing Countries: The Case of Turkey, Tunisia and Jordan”, European Journal of Economics, Finance and Administrative Sciences, 16, 84-98.

Al-Maadid A. \& M.G. Caporale \& F. Spagnolo (2017), "Spillovers between food and energy prices and structural breaks", International Economics, 150, 1-18.

Arouri, M. \& J. Jouini \& D.K. Nguyen (2011), "Volatility spillovers between oil prices and stock sector returns: Implications for portfolio management", Journal of International Money and Finance, 30, 1387-1405. 
Abioğlu, V. (2021), "Volatility Spillovers and Correlations between Oil Prices and Stock Sectors in Turkey: Implications on Portfolio Hedging and Diversification Opportunities", Sosyoekonomi, 29(47), 79-106.

Arouri, M. \& J. Jouini \& D.K. Nguyen (2012), “On the impacts of oil price fluctuations on European equity markets: Volatility spillover and hedging effectiveness", Energy Economics, 34, 611-617.

Baillie, R.T. \& R.J. Myers (1991), "Bivariate GARCH estimation of the optimal commodity futures hedge", Journal of Applied Econometrics, 6, 109-124.

Basher, A.S. \& P. Sadorsky (2006), "Oil Price Risk and Emerging Stock Markets", Global Finance Journal, 17, 224-251.

Basher, A.S. \& P. Sadorsky (2016), "Hedging emerging market stock prices with oil, gold, VIX, and bonds: a comparison between DCC, ADCC and GO-GARCH”, Energy Economics, 54, 235-247.

Benet, B.A. (1992), "Hedge period length and ex ante futures hedging effectiveness: the case of foreign exchange risk cross hedges", Journal of Futures Markets, 12, 163-175.

Büberkökü, Ö. (2017), "Examining the Impact of the Oil Prices on the Turkish Stock Market under Multiple Structural Breaks”, Bankacılık ve Sermaye Piyasası Araştırmaları DergisiBSPAD, 1(2), 15-32.

Caporale, G.M. \& F.M. Ali \& N. Spagnolo (2015), "Oil price uncertainty and sectoral stock returns in China: A time-varying approach”, China Economic Review, 34, 311-321.

Cappiello, L. \& R.F. Engle \& K. Sheppard (2006), “Asymmetric Dynamics in the Correlations of Global Equity and Bond Returns", Journal of Financial Econometrics, 4(4), 537-572.

Chang, C. \& M. McAleer \& R. Tansuchat (2011), "Crude oil hedging strategies using dynamic multivariate GARCH”, Energy Economics, 33, 912-923.

Ederington, L.H. (1979), "The hedging performance of the new futures markets”, The Journal of Finance, 34, 157-170.

Engle, R.F. (2002), "Dynamic conditional correlation: A simple class of multivariate generalized autoregressive conditional heteroskedasticity models", Journal of Business and Economic Statistics, 20(3), 339-350.

Eyüboğlu, K. \& S. Eyüboğlu (2016), "Examining the Relationship among the Natural Gas, Oil Prices and Sub-Indexes of BIST-Industrial”, Journal of Yasar University, 11(42), 150-162.

Figlewski, S. (1985), "Hedging with stock index futures: estimation and forecasting with error correction model", Journal of Futures Markets, 13, 743-752.

Gencer, G.H. \& S. Demiralay (2014), "Shock and Volatility Spillovers between Oil Prices and Turkish Sector Returns", International Journal of Economics and Finance, 6(2), 174180.

Gogineni, S. (2010), "Oil and the stock market: An industry level analysis", The Financial Review, 45, 995-1010.

Gönüllü, Ç.O. \& E. Otluoğlu \& M.H. Şengöz (2015), "The Impact of Crude Oil Price Changes on Petrochemical Industry Returns", International Journal of Economic and Administrative Studies, 14, 223-234.

Güler, S. \& R. Tunç \& Ç. Orçun (2010), "Petrol Fiyat Riski ve Hisse Senedi Fiyatları Arasındaki İlişkinin Belirlenmesi: Türkiye'de Enerji Sektörü Üzerinde Bir Uygulama”, Atatürk Üniversitesi İktisadi ve İdari Bilimler Dergisi, 24(4), 297-315.

Guo, H. \& L. Kliesen (2005), "Oil price volatility and US macroeconomic activity”, Federal Reserve Bank of St. Louis Review, 87, 669-683. 
Abioğlu, V. (2021), "Volatility Spillovers and Correlations between Oil Prices and Stock Sectors in Turkey: Implications on Portfolio Hedging and Diversification Opportunities", Sosyoekonomi, 29(47), 79-106.

Hammoudeh, S. \& Y. Yuan \& M. McAleer \& M. Thompson (2010), "Precious metals-exchange rate volatility transmissions and hedging strategies", International Review of Economics and Finance, 19, 633-647.

Huang, R.D. \& R.W. Masulis \& H.R. Stoll (1996), "Energy shocks and financial markets”, Journal of Futures Markets, 16, 1-27.

İşcan, E. (2010), “The Impact of Oil Prices on Stock Prices”, Maliye Dergisi, 158, 607-617.

Jones, C.M. \& G. Kaul (1996), “Oil and the stock markets”, Journal of Finance, 51, 463-491.

Kang, S.H. \& R. Mclver \& S.M. Yoon (2017), "Dynamic spillover effects among crude oil, precious metal and agricultural commodity futures markets", Energy Economics, 62, 19-32.

Kapusuzoglu, A. (2011), "Relationships between Oil Price and Stock Market: An Empirical Analysis from Istanbul Stock Exchange (ISE)", International Journal of Economics and Finance, 3(6), 99-106.

Kirkulak-Uludag, B. \& O. Safarzadeh (2018), "The interactions between OPEC oil price and sectoral stock returns: Evidence from China”, Physica A, 508, 631-641.

Kroner, K.F. \& J. Sultan (1993), "Time-Varying Distributions and Dynamic Hedging with Foreign Currency Futures”, Journal of Financial and Quantitative Analysis, 28(4), 535-551.

Kroner, K.F. \& V.K. Ng (1998), "Modeling Asymmetric Comovement of Asset Returns”, The Review of Financial Studies, 11(4), 817-844.

Ku, Y.H. \& H-C. Chen \& K-H. Chen (2007), "On the application of the dynamic conditional correlation model in estimating optimal time varying hedge ratios", Applied Economics Letters, 14(7), 503-509.

Lee, K. \& S. Ni (2002), "On the dynamic effects of oil shocks: A study using industry level data", Journal of Monetary Economics, 49, 823-852.

Lin, B. \& P.K. Wesseh \& M.O. Appiah (2014), "Oil price fluctuation, volatility spillover and the Ghanian equity market: Implication for portfolio management and hedging effectiveness", Energy Economics, 42, 172-182.

Ling, S. \& M. McAleer (2003), "Asymptotic theory for a vector ARMA-GARCH model”, Econometric Theory, 19, 278-308.

Malik, F. \& B.T. Ewing (2009), "Volatility transmission between oil prices and equity sector returns", International Review of Financial Analysis, 18, 95-100.

Malik, S. \& S. Hammoudeh (2007), "Shock and volatility transmission in the oil, US and Gulf equity markets", International Review of Economics and Finance, 17, 357-368.

McAleer, M. \& S. Hoti \& F. Chan (2009), "Structure and Asymptotic Theory for Multivariate Asymmetric Conditional Volatility”, Econometric Reviews, 28(5), 422-440.

Mensi, W. \& M. Beljid \& A. Boubaker \& S. Managi (2013), "Correlations and volatility spillovers across commodity and stock markets: linking energies, food, and gold", Economic Modelling, 32, 15-22.

Mensi, W. \& S. Hammoudeh \& D.K. Nguyen \& S-M. Yoon (2014), "Dynamic spillovers among major energy and cereal commodity prices", Energy Economics, 43, 225-294.

Myers, R.J. \& S.R. Thompson (1989), “Generalized optimal hedge ratio estimation”, American Journal of Agricultural Economics, 71, 858-867.

Park, J. \& R.A. Ratti (2008), "Oil price shocks and stock markets in the US and 13 European countries", Energy Economics, 30, 2587-2608. 
Ross, S. (1989), "Information and volatility: the no-arbitrage martingale approach to timing and resolution irrelevancy", Journal of Finance, 44, 1-17.

Sadorsky, P. (2012), "Correlations and volatility spillovers between oil prices and the stock prices of clean energy and technology companies", Energy Economics, 34, 248-255.

Sayılgan, G. \& C. Süslü (2011), "Makroekonomik Faktörlerin Hisse Denedi Getirilerine Etkisi: Türkiye ve Gelişmekte Olan Piyasalar Üzerine Bir İnceleme”, BDDK Bankacılık ve Finansal Piyasalar Dergisi, 5(1), 73-96.

Şener, S. \& V. Yılancı \& M. Tıraşoğlu (2013), “Analyzing the Hidden Cointegration between Oil Prices and Stock Prices", The Journal of Social Economic Research, 13(26), 231-248.

Soytas, U. \& A. Oran (2011), "Volatility spillover from world oil spot markets to aggregate and electricity stock index returns in Turkey", Applied Energy, 88, 354-360.

Unlu, U. \& M. Topcu (2012), "Do Oil Prices Directly Affect Stock Markets: Evidence from Istanbul Stock Exchange”, Iktisat, Isletme ve Finans, 27(319), 75-88. 